\title{
Numerical study of a multiple-segment metal foam-PCM latent heat storage unit: effect of porosity, pore density and location of heat source
}

\author{
Pouyan Talebizadeh Sardari ${ }^{1}$, Hayder I. Mohammed ${ }^{1,2}$, Donald Giddings ${ }^{1}$, Gavin S. walker ${ }^{3}$, \\ Mark Gillott ${ }^{4}$, David Grant ${ }^{3}$ \\ ${ }^{1}$ Department of Physics, Collage of Education, University of Garmian, Kurdistan, Iraq. \\ ${ }^{2}$ Fluids and Thermal Engineering Research Group, Faculty of Engineering, the University of \\ Nottingham, University Park, Nottingham NG7 2RD, UK. \\ ${ }^{3}$ Advanced Materials Research Group, Faculty of Engineering, the University of Nottingham, \\ University Park, Nottingham NG7 2RD, UK. \\ ${ }^{4}$ Buildings, Energy and Environment Research Group, Faculty of Engineering, the University of \\ Nottingham, University Park, Nottingham NG7 2RD, UK.
}

\begin{abstract}
This study numerically investigates the performance of the melting process for a PCM based heat storage system under the effect of different variables in a vertical container with a copper metal foam. Different cases were studied and compared including the effects of variable porosities and pore densities, non-equilibrium porous medium model, a multiple-segment metal foam case and different heater locations in the system on the liquid fraction and temperature as presented by contour plots and diagrams. The results show high performance for the copper foam-PCM unit compared with on its own PCM, for reducing the melting time by almost $85 \%$. By changing the location of constant temperature heater from the bottom to the side and top surface, the melting time decreases by $70.5 \%$ and $4.7 \%$, respectively. By using a multiple-segment porous system, the melting time reduces by $3.5 \%$ compared with the case of uniform porosity. Furthermore, the more accurate non-equilibrium numerical model shows a $7.4 \%$ difference in the melting time compared with the equilibrium model. This study optimises the design to improve practical application performance and to reduce waste energy.
\end{abstract}


Keywords: Phase change materials; Latent heat storage; Multiple-segment porous medium; Melting; Non-equilibrium thermal model.

\begin{tabular}{|c|c|c|c|}
\hline \multicolumn{4}{|c|}{ Nomenclature } \\
\hline$A_{m}$ & Mushy zone constant & $t_{m}$ & Melting/solidification time (s) \\
\hline$C$ & Inertial coefficient of porous medium & $T$ & Temperature $(\mathrm{K})$ \\
\hline$C_{p, f}$ & Specific heat of PCM (J/kgK) & $T_{r e f}$ & Reference temperature $(\mathrm{K})$ \\
\hline$c_{p, s}$ & Specific heat of solid $(\mathrm{J} / \mathrm{kgK})$ & $\vec{V}$ & Velocity vector $(\mathrm{m} / \mathrm{s})$ \\
\hline$c_{p, f}$ & Specific heat of fluid $(\mathrm{J} / \mathrm{kgK})$ & & \\
\hline$d_{p}$ & Pore size $(\mathrm{m})$ & \multicolumn{2}{|c|}{ Greek symbols } \\
\hline$d_{l}$ & Ligament diameter of the porous medium (m) & $\beta_{f}$ & Thermal expansion coefficient $(1 / \mathrm{K})$ \\
\hline$g$ & Gravitational acceleration $\left(\mathrm{m} / \mathrm{s}^{2}\right)$ & $\varepsilon$ & Porosity \\
\hline$h$ & Sensible enthalpy $(\mathrm{J} / \mathrm{kg})$ & $\lambda$ & Liquid fraction \\
\hline$h_{\text {ref }}$ & Sensible enthalpy at reference temperature $(\mathrm{J} / \mathrm{kg})$ & $\mu_{f}$ & Dynamic viscosity (kg/ms) \\
\hline$H$ & Total enthalpy $(\mathrm{J} / \mathrm{kg})$ & $\rho_{f}$ & Density $\left(\mathrm{kg} / \mathrm{m}^{3}\right)$ \\
\hline$k_{e}-k_{e f f}$ & Effective thermal conductivity (W/mK) & $\rho_{f, r e f}$ & Density at reference temperature $\left(\mathrm{kg} / \mathrm{m}^{3}\right)$ \\
\hline$k_{f e}$ & Thermal conductivity of fluid (PCM) (W/mK) & $\omega$ & Pore density (PPI) \\
\hline$k_{s e}$ & $\begin{array}{l}\text { Solid Thermal conductivity (porous material) } \\
(\mathrm{W} / \mathrm{mK})\end{array}$ & $\Delta H$ & Latent heat $(\mathrm{J} / \mathrm{kg})$ \\
\hline$K$ & Permeability of porous medium $\left(\mathrm{m}^{2}\right)$ & $\Delta P$ & Pressure drop $(\mathrm{Pa})$ \\
\hline$L_{f}$ & Latent heat of fusion $(\mathrm{J} / \mathrm{kg})$ & & \\
\hline$m_{p c m}$ & Mass of PCM (kg) & \multicolumn{2}{|c|}{ Subscripts } \\
\hline$m_{\text {por }}$ & Mass of porous medium $(\mathrm{kg})$ & $f$ & Fluid (PCM) \\
\hline$P$ & Pressure $(\mathrm{Pa})$ & $s$ & Solid (metal foam) \\
\hline$\vec{S}$ & Source term in momentum equation & ref & Reference \\
\hline$S_{L}$ & Source term in energy equation & & \\
\hline
\end{tabular}

\section{Introduction}

Fossil fuel is the main source of the $10^{18} \mathrm{~J}$ of energy consumed daily in the world. This fuel has two main negative impacts, polluting emissions to the environment, and wasting heat due to low-efficiency systems. The temperatures which are released to the environment vary between $35^{\circ} \mathrm{C}$ in many power plants to $1600^{\circ} \mathrm{C}$ in some ironmaking factories. Effective energy 
storage is required to solve some environmental concerns and to find advantages from the wasted heat in addition to the other applications of thermal energy storage [1].

There are different types of thermal storage materials used depending on their features and the function of usage. Phase change materials (PCMs), which consider as latent heat thermal energy storage materials, release or absorb heat during the phase change process [2]. PCMs have been examined in construction field [3, 4], industrial waste heat recovery [5], solar heat source systems [6-8] and the thermal stability of PCMs have been investigated by repeating thermal cycle tests $[9,10]$. The disadvantage of the PCMs is presented as a low thermal conductivity (around $0.2 \mathrm{~W} / \mathrm{m} \mathrm{K}$ for most paraffin [11]), which causes a low power density and slow charging/discharging rate. Several studies have considered solving this issue by studying the thermophysical properties of PCMs [12-14]; others are interested in improving the efficiency of the related heat exchangers [15-19].

Heat transfer efficiency in PCM can be improved via various techniques; high thermal conductivity extended surfaces [20-23]; mixing PCM with high thermal conductivity materials, such as expanded graphite [24-26] or silicon carbide ceramic [27]; heat pipes [28, 29]; metal foam or matrix [30-33]; nanomaterial technology [34-37]. Metal foam is considered as one of the best techniques to enhance the heat transfer in PCMs as reported by Mahdi and Nsofor [22] who studied numerically the heat transfer enhancement in the PCM using multiple-segment metal foam. They suggested that multiple-segment metal foam, following in the heat flow directions, is useful to provide a uniform temperature distribution. In a separate study [22], they investigated the effects of the nanoparticles and fins addition on the solidification rate of PCMs and found that using fins improves the phase change rate better than alone nanoparticles or the combination of both.

There are some evidence of phase change process through a system filled with a porous medium or metal foam. The effect of the porosity of Aluminium metal foam on the latent heat 
storage (LHS) device was experimentally investigated by Atal et al. [30]. They found that the lower porosity could make the phase change cycle time shorter due to the consequent amount of high conductive metal foam. Zhao et al. [38] studied the effects of the copper metal foam on the phase change rate of RT58 and stated that the metal foam can enhance the phase change rate around 10 times depending on the material and the conditions used. Xu et al. [39] studied numerically the PCM thermal energy storage using a porous medium with partial filling. The results showed that when the porous medium located on the lower part of the PCM, the thermal conductivity increased, which reduced the melting time and gave constant melting rate. Lafdi et al. [40] experimentally studied the influence of porosity and pore size of metal (Al) foam on the melting rate of paraffin. They found that the temperature can achieve a steady state quicker by using higher porosity and pore size of the metal foam. Nithyanandam and Pitchumani [41] numerically studied the effect of metal foam on LHS system in heat pipes. They found that lower pore size improves the rate of heat transfer. The rate of energy transfer of the LHS system worked with pure molten salt was studied experimentally and numerically by Zhang et al. [42]. They found that the natural convection heat transfer reduces by using metal foam. Yang et al. [43] and Zhu et al. [44] stated that using multiple-metal foam sections could enhance the storage efficiency of the PCMs.

Accelerating the phase change process causes a higher natural convection heat transfer as well as providing a cost-effective consideration by saving some PCM or reducing the power applied to the system. Tao et al. [45] experimentally investigated the influence of the phase change rate efficiency of the PCM in a horizontal pipe. They stated that increasing the area of the natural convection reduces the melting time of the PCM. Wang et al. [46] simulated the phase change process of the PCM in sleeve-pipes and compared it with the effect of the fin shape tube. They found that by considering the natural convection, the effective angle of the tubes was between $60^{\circ}-90^{\circ}$ from the horizontal. Tao et al. [47] investigated the performance of an energy storage 
system under the effect of natural convection. They found that adding fins to the tube accelerates the melting rates and makes the melting more uniform on the boundary.

All the presented investigations studied one side of the PCM phase change such as studying the effect of the porous medium or the fins, nanoparticles, porosity and the pore size. Therefore, the authors found that studying different aspects of one system is important to understand how different variables affects the performance of the phase change process. The current work numerically investigates the melting process performance in a symmetric square container filled with copper foam impregnated with paraffin wax. Heat transfer rate, average temperature and velocity caused by natural convection are studied and compared in various cases including effects of porosity, pore size, non-equilibrium porous medium, multiple-segment porous system and different heater locations in the system. The current study provides guidance for good design practice and optimizes the conditions that could apply to similar PCM storage systems. This study also aims to develop the possibility of practical application for highperformance LHS units using metal foam-PCM composite.

\section{Mathematical modelling}

The principle of equalizing the porosity and the liquid fraction which is called the enthalpyporosity model was considered in the numerical approach of the melting process. The pressure drop is accounted for the momentum equation due to viscous and inertial losses, which caused by the porous medium and the solidification state [48]. The numerical assumptions $[49,50]$ :

- An incompressible and Newtonian fluid is assumed for the liquid phase.

- Boussinesq approximation is applied to consider the effect of natural convection due to the small temperature gradient in the field.

- The porous medium is assumed homogeneous and isotropic. 
- The volume expansion of the PCM is neglected during phase change due to considering a close system.

- Viscous dissipation is negligible.

Then, the governing equations are given as [42]:

Continuity:

$\frac{\partial \rho_{f}}{\partial t}+\nabla \cdot \rho_{f} \vec{V}=0$

Momentum:

$\frac{\rho_{f}}{\varepsilon} \frac{\partial \vec{V}}{\partial t}+\frac{\rho_{f}}{\varepsilon^{2}}(\vec{V} \cdot \nabla) \vec{V}=-\nabla P+\frac{\mu_{f}}{\varepsilon}\left(\nabla^{2} \vec{V}\right)-\rho_{f, r e f} \beta_{f} \varepsilon\left(T-T_{r e f}\right) \vec{g}-\vec{S}-\vec{F}$

Both thermal models of non-equilibrium and equilibrium are considered in this study for the energy equation as described in the ANSYS FLUENT theory guide. Energy equation for the equilibrium model, in which the porous medium and PCM in each cell have the same temperature is given as:

$$
\frac{\partial \varepsilon \rho_{f} C_{p, f} T}{\partial t}+\nabla\left(\rho_{f} C_{p, f} \vec{V} T\right)=\nabla\left(k_{e} \nabla T\right)-S_{L}
$$

Energy equation for non-equilibrium model, in which the porous medium and PCM establish heat transfer between them is given as:

For the PCM:

$$
\frac{\partial \varepsilon \rho_{f} C_{p, f} T_{f}}{\partial t}+\nabla\left(\rho_{f} C_{p, f} \vec{V} T_{f}\right)=\nabla\left(k_{e} \nabla T_{f}\right)-S_{L}-h_{s f} A_{s f}\left(T_{f}-T_{s}\right)
$$

For the porous medium:

$$
(1-\varepsilon) \rho_{s} C_{p, s}\left(\frac{\partial T_{s}}{\partial t}\right)=\nabla\left(k_{s e} \nabla T_{s}\right)-h_{s f} A_{s f}\left(T_{s}-T_{f}\right)
$$

In the thermal equilibrium model, volume average thermal conductivity is assumed between the PCM and the porous medium as [50]:

$$
k_{e}=(1-\varepsilon) k_{s}+\varepsilon k_{f}
$$


In the thermal non-equilibrium model, the effective thermal conductivity of the fluid and solid should be determined and employed independently. There are different theoretical models in the literature based on the porous characteristics, the thermal conductivity of solid and fluid ${ }^{[51-}$ 55]. The models are classified based on the unit cell. The 'tetrakaidecahedron' cell model of Boomsma and Poulikakos is introduced in this study which is first introduced in 2001 [52] and then corrected in 2011 [56]. In this model, the effective thermal conductivity is defined as [52, 56]:

$$
k_{e f f}=\frac{1}{\sqrt{2}\left(R_{A}+R_{B}+R_{C}+R_{D}\right)}
$$

where

$$
\begin{aligned}
& R_{A}=\frac{4 \sigma}{\left(2 e^{2}+\pi \sigma(1-e)\right) k_{s}+\left(4-2 e^{2}-\pi \sigma(1-e)\right) k_{f}} \\
& R_{B}=\frac{(e-2 \sigma)^{2}}{(e-2 \sigma) e^{2} k_{s}+\left(2 e-4 \sigma-(e-2 \sigma) e^{2}\right) k_{f}} \\
& R_{C}=\frac{\sqrt{2}-2 e}{\sqrt{2} \pi \sigma^{2} k_{s}+\left(2-\sqrt{2} \pi \sigma^{2}\right) k_{f}} \\
& R_{D}=\frac{2 e}{e^{2} k_{s}+\left(4-e^{2}\right) k_{f}}
\end{aligned}
$$

where $e=0.16$ and

$$
\sigma=\sqrt{\frac{\sqrt{2}\left(2-\left(\frac{3 \sqrt{2}}{4}\right) e^{3}-2 \varepsilon\right)}{\pi(3-2 \sqrt{2} e-e)}}
$$

and

$$
\begin{aligned}
& k_{f e}=k_{e f f} \mid k_{s=0} \\
& k_{s e}=k_{e f f} \mid k_{f=0}
\end{aligned}
$$

To calculate the local heat transfer between the porous medium and PCM, the porous structure is usually considered as cylinders and the laminar flow of liquid PCM in porous structure is 
considered similar to the flow around a cylinder. Therefore, in Eqs. (4-5), the interstitial heat transfer coefficient is calculated as $[42,57]$ :

$$
h_{s f}=\left\{\begin{array}{cc}
0.76 \operatorname{Re}_{d}^{0.4} \mathrm{Pr}^{0.37} k_{p c m} / d_{l} \text { for } & 0<R e_{d} \leq 40 \\
0.52 \operatorname{Re}_{d}^{0.5} \operatorname{Pr}^{0.37} k_{p c m} / d_{l} \text { for } & 40<R e_{d} \leq 1000 \\
0.26 \operatorname{Re}_{d}^{0.6} \operatorname{Pr}^{0.37} k_{p c m} / d_{l} \text { for } & 1000<R e_{d} \leq 20000
\end{array}\right.
$$

where

$$
R e_{d}=\rho_{p c m} \sqrt{u^{2}+v^{2}} d_{l} /\left(\varepsilon \mu_{p c m}\right)
$$

and $A_{s f}$ is the specific surface area of the porous medium given as:

$$
A_{s f}=\frac{3 \pi d_{l}\left(1-e^{-(1-\varepsilon) / 0.04}\right)}{0.59 d_{p}^{2}}
$$

The source terms in the momentum equation are accounted for the pressure loss due to the influence of the melting process, which are defined according to the Darcy's law of damping as [49]:

$$
\vec{S}=A_{m} \frac{(1-\lambda)^{2}}{\lambda^{3}+0.001} \vec{V}
$$

It should be noted that the porosity in the governing equations for PCM only case is equal to 1 and the momentum equation lost the source terms due to the presence of porous media. Additionally, $\lambda$ is defined as [58]:

$$
\lambda=\frac{\Delta H}{L_{f}}=\left\{\begin{array}{cc}
0 & \text { if } T<T_{\text {Solidus }} \\
1 & \text { if } T>T_{\text {Liquidus }} \\
\frac{T-T_{\text {Solidus }}}{T_{\text {Liquidus }}-T_{\text {Solidus }}} & \text { if } T_{\text {Solidus }}<T<T_{\text {Liquidus }}
\end{array}\right\}
$$

where $\Delta H$ may vary between zero for solid and $L_{f}$ for liquid.

The total enthalpy can calculated as:

$$
H=h+\Delta H
$$

where $h$ is defined as follows: 


$$
h=h_{r e f}+\int_{T_{r e f}}^{T} C p_{f} d T
$$

The last term on the right-hand side of the momentum equation, which caused by the porous medium is defined as:

$$
\vec{F}=\left(\frac{\mu_{f}}{K}+\frac{\rho_{f} C|\vec{V}|}{\sqrt{K}}\right) \vec{V}
$$

where $|\vec{V}|$ is the magnitude of the velocity vector. In the above equations, the first term is the viscous loss term and the second term is the inertial loss term. In these equations, $K$ and $C$ are given as [48]:

$$
\begin{aligned}
& K=0.00073 d_{p}^{2}(1-\varepsilon)^{-0.224}\left(\frac{d_{l}}{d_{p}}\right)^{-1.11} \\
& C=0.00212(1-\varepsilon)^{-0.132}\left(\frac{d_{l}}{d_{p}}\right)^{-1.63}
\end{aligned}
$$

where $d_{l}$ is obtained as $[18,32,50]$ :

$$
d_{l}=1.18 d_{p} \sqrt{\frac{1-\varepsilon}{3 \pi}}\left(\frac{1}{1-e^{-(1-\varepsilon) / 0.04}}\right)
$$

and $d_{p}$ is calculated as:

$$
d_{p}=0.0254 / \omega(\mathrm{PPI})
$$

where $\omega$ is defined in terms of PPI means the number of pores per inch.

The source term in the energy equation is given as [59]:

$$
S_{L}=\frac{\partial \varepsilon \rho_{f} \lambda L_{f}}{\partial t}+\nabla\left(\rho_{f} \vec{V} \lambda L_{f}\right)
$$

\section{Geometry and boundary conditions:}

The LHS containment is a rectangular box with the dimensions of $5 \mathrm{~cm} \times 5 \mathrm{~cm} \times 15 \mathrm{~cm}$ filled firstly with copper foam and PCM and then with PCM only. Due to the symmetric condition 
of the problem, only one-quarter of the system is modelled using the symmetry boundary condition which is also assumed in different studies in the literature $[49,50,60]$. Apart from the heated surface, all the other walls are considered adiabatic to eliminate the effect of heat loss. The geometry, as well as the boundary conditions, are shown in Fig. 1 which shows different heater locations considered. The initial temperature of the PCM is $292 \mathrm{~K}$. Note that for all the porous cases, the dimensions of the system (in $\mathrm{x}$ and $\mathrm{z}$-directions) are enhanced to have the same mass of the PCM in order to have an equal heat storage capacity for all studied systems which makes the comparison more straightforward.

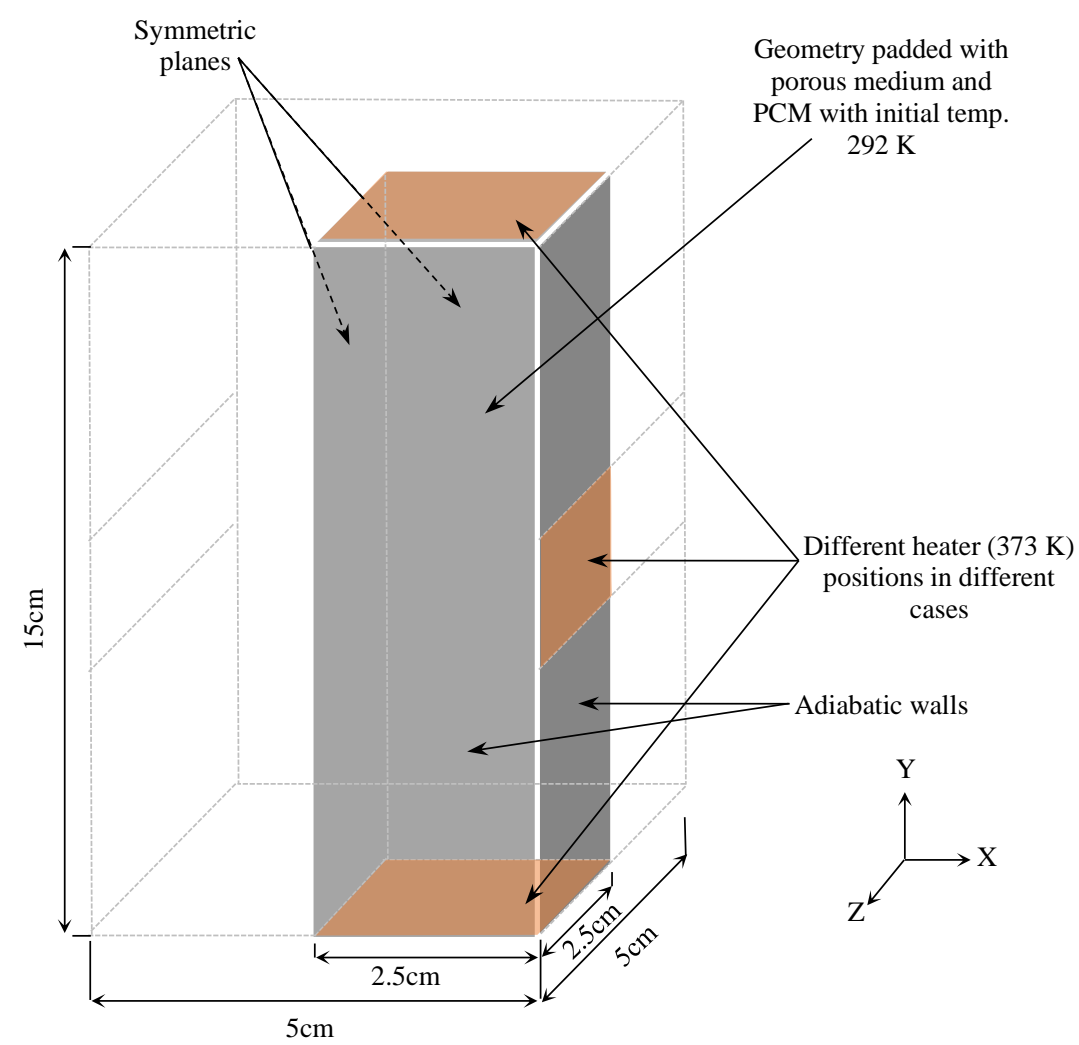

Fig. 1. The schematic of the studied geometry (not in scale)

RT-35 (RUBITHERM) is considered as the PCM because it has a relatively low melting temperature $\left(35^{\circ} \mathrm{C}\right)$ comparing to the other materials. The physical properties of RT-35 presented in Table 2 . 
Table 2 Physical properties of RT 35 [36]

\begin{tabular}{cc}
\hline Property & RT35 \\
\hline Liquidus temperature $\left({ }^{\circ} \mathrm{C}\right)$ & 309 \\
Solidus temperature $\left({ }^{\circ} \mathrm{C}\right)$ & 302 \\
Heat of fusion $(\mathrm{kJ} / \mathrm{kg})$ & 170 \\
Specific heat $(\mathrm{kJ} / \mathrm{kg} . \mathrm{K})$ & 2 \\
Density $\left(\mathrm{kg} / \mathrm{m}^{3}\right)$ & 815 \\
Thermal conductivity $(\mathrm{W} / \mathrm{m} . \mathrm{K})$ & 0.2 \\
Viscosity $($ Pa.s $)$ & 0.023 \\
Thermal expansion coefficient $(1 / \mathrm{K})$ & 0.001 \\
\hline
\end{tabular}

\section{Numerical process and validation}

The governing equations of this process are solved by ANSYS-FLUENT with the aid of UserDefined Functions (UDF) for calculating the interfacial heat transfer coefficient between the PCM and the metal foam in the porous case in the thermal non-equilibrium model as described in section 2. The governing equations are discretised with a double precision solver using the SIMPLE algorithm. PRESTO pressure interpolation scheme is used due to buoyancy, while the quadratic upwind discretisation, QUICK scheme is employed for the momentum and energy equations, both for enhancing the accuracy of the numerical method. The values for under-relaxation factors are set to $0.3,0.3,0.8$ and 0.5 for the pressure, velocity, energy, and the liquid fraction, respectively. The convergence criteria for continuity, momentum and energy equations are set to $10^{-4}, 10^{-4}$ and $10^{-6}$, respectively.

For the mesh independency analysis, a different number of cells are studied presented in Table 2 for different numbers of cells in the y-direction. Note that 25 cells are considered for both $\mathrm{x}$ and z-direction. Due to the natural convection in the y-direction, a higher number of cells is applied in the y-direction. As shown, after the cell number of 60, increasing the number of cells has no considerable effect on the results and therefore the number of cells is considered 60 for 
further analysis. The size of the time step is considered $0.5 \mathrm{~s}$ and $0.25 \mathrm{~s}$ for the cases of porousPCM and PCM only, respectively.

Table 2. Mesh independency analysis, a different number of cells

$\begin{array}{ccccccc}\begin{array}{c}\text { Number of } \\ \text { cells in Y } \\ \text { direction }\end{array} & \begin{array}{c}\text { Melting } \\ \text { time }(\mathrm{s})\end{array} & \begin{array}{c}\text { Average temperature } \\ \text { with total converted } \\ \text { phase }(\mathrm{K})\end{array} & \begin{array}{c}\text { Average } \\ \text { temperature } \\ \text { after } 1000 \mathrm{~s}(\mathrm{~K})\end{array} & \begin{array}{c}\text { Heat flux in total } \\ \text { phase change } \\ (\mathrm{W} / \mathrm{m} 2)\end{array} & \begin{array}{c}\text { Heat flux in } \\ (\mathrm{W} / \mathrm{m} 2)\end{array} & \begin{array}{c}\text { Time of } \\ \text { simulation for }\end{array} \\ 40 & 737 & 335.06 & 350.22 & 13.72 & 8.14 & 12.5 \\ 50 & 740 & 335.71 & 351.15 & 13.85 & 8.42 & 14 \\ \mathbf{6 0} & \mathbf{7 4 5} & \mathbf{3 3 7 . 2 6} & \mathbf{3 5 2 . 9 7} & \mathbf{1 4 . 1} & \mathbf{8 . 9 8} & \mathbf{1 5 . 4} \\ 70 & 745 & 337.52 & 353.37 & 14.15 & 9.1 & 17.2 \\ 80 & 746 & 337.46 & 353.26 & 14.13 & 9.06 & 19\end{array}$

The mushy-zone constant is selected from the literature that performed a CFD simulation using ANSYS FLUENT software. $A_{m}$ is defined in the range of $10^{4}-10^{7} \mathrm{~kg} / \mathrm{m} 3 \mathrm{~s}$ which should be determined based on an experimental study and varied with the geometrical and operating conditions of the system. $A_{m}$ is an important parameter for the effect of natural convection after the presence of liquid PCM in the domain. However, in the presence of the porous medium, due to the small effect of natural convection, $A_{m}$ has a small effect on the flow distribution. For the sensitivity analysis, three different values of $10^{4}, 10^{5}$ and $10^{6}$ are examined for $A_{m}$ and the results show less than $0.5 \%$ difference on the melting time for different cases. Therefore, as recommended in many studies in the literature, $A_{m}$ is considered equal to $10^{5}$ [61-63].

The schematics of the entire computational mesh, as well as the front view and top view, are shown in Fig. 2. Note that a finer mesh is employed near the walls due to the formation of the thermal boundary layer in this area. 

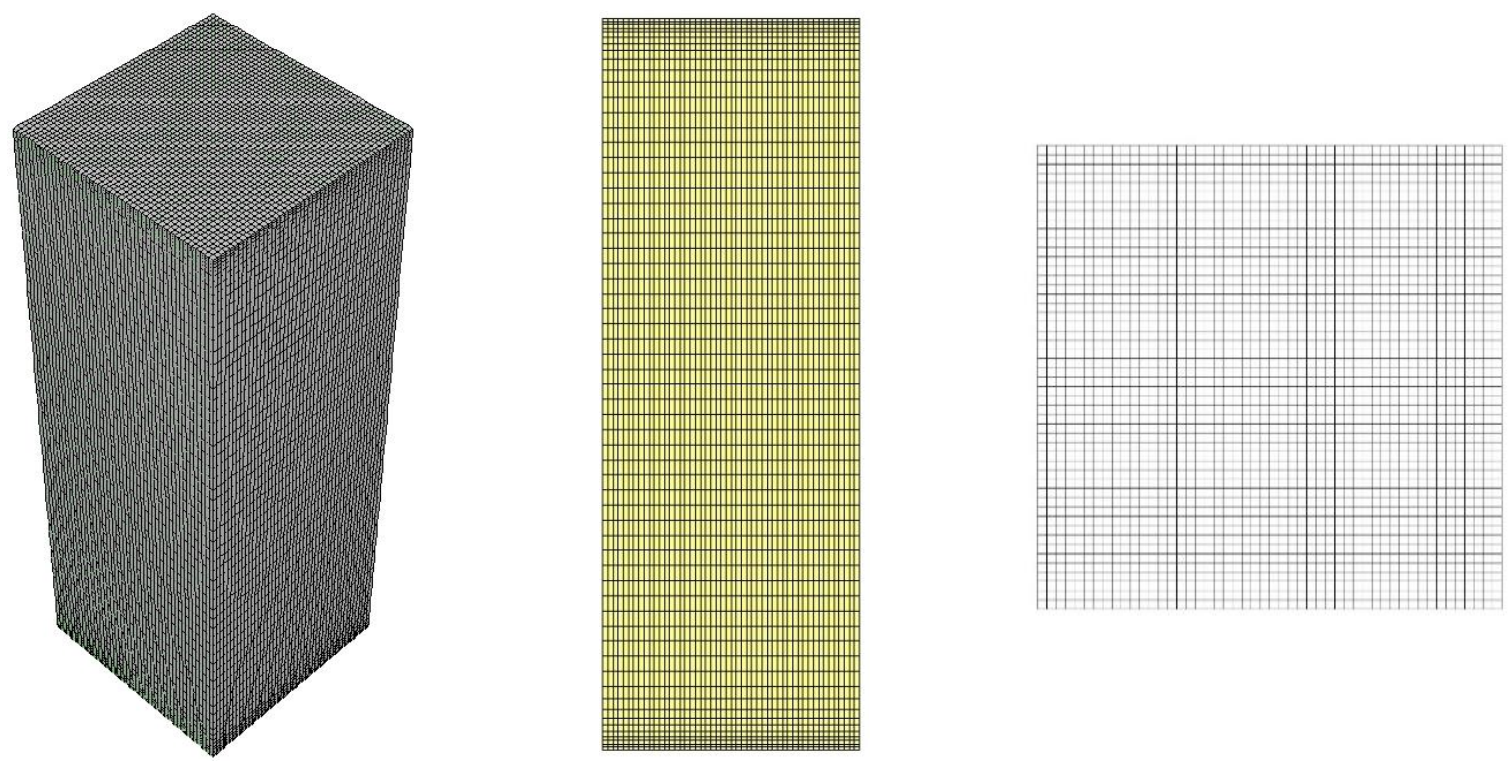

Fig. 2. The computational domain at different views

To validate the code, the experimental results of Zhao et al. $[11,38]$ are used for comparison using RT-58 PCM with a porous medium (porosity of 95\% and pore density of 10 pores per inch (PPI)). The studied geometry is a rectangular enclosure with a heat flux boundary condition from the bottom and free convection for the external walls. The results of the temperature at the centre at the height of $8 \mathrm{~mm}$ from the bottom are presented in Fig. 3 and an excellent agreement can be found between different studies. As shown, the thermal equilibrium model is not capable to simulate the PCM embedded in the porous medium very well especially after the liquidus temperature. 


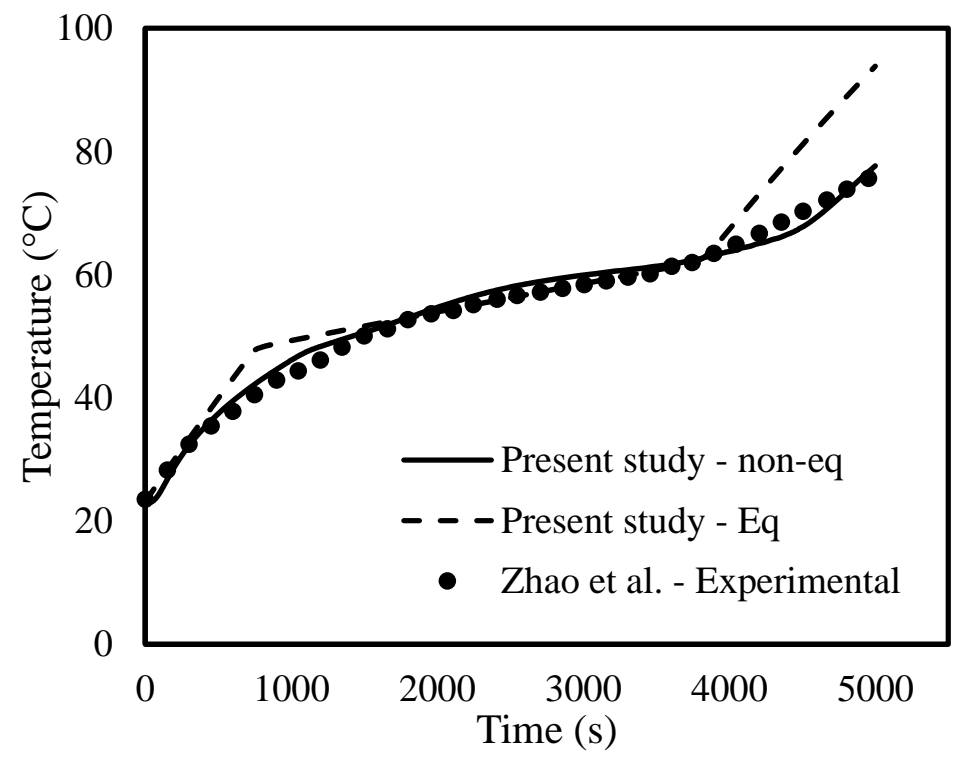

Fig. 3. The validation results of porous-PCM in compare with different numerical and experimental studies in the literature $[11,50]$

\section{Results and discussion}

In this study, after comparing the results of the porous-PCM composite case with the PCM only, the effects of porosity and pore density are studied. Then, the influence of the thermal non-equilibrium model of the porous-PCM is compared with the equilibrium model and at last the effect of the location of the heater and the multiple-segment of the porous medium are investigated.

\subsection{Effect of the presence of a high conductivity metal foam}

For the PCM only, initially, the heat transfers by conduction in the solid mass of the PCM, after the melting process, the heat transfers via convection and conduction. To increase the heat transfer inside the PCM, a porous foam is prepared and the PCM is injected into the porous medium results in the enhancement of heat transfer rate in the domain. Therefore, the heat is transferred from the heater faster in the entire domain which reduces melting time. Fig. 4 illustrates the contour plot of liquid fraction for the cases of porous-PCM with the porosity of 
$90 \%$ and pore density of 30PPI and PCM only, respectively, at different times. A higher amount of PCM liquid fraction can be seen for the case of porous-PCM due to the higher rate of heat transfer in the domain. In the porous-PCM unit, the heat is transferred by a conduction mechanism through the high conductivity metal porous medium in the PCM only. However, in the PCM only system, natural convection gradually becomes dominant for transferring heat. After 800s, all the PCM melts in the case of porous-PCM; however, the liquid fraction is only 0.174 for the case of PCM only.

Fig. 5 shows the contour plot of temperature at different times for the systems of porous-PCM compared with the PCM only system. Higher temperature for the porous-PCM case can be seen compared with the PCM only case. Due to the domination of natural convection in the PCM case, the Benard formation, which defines as natural convection, happening in a horizontal layer of fluid heated from below. However, for the porous-PCM, just the conduction heat transfer can be seen causes uniform layers of PCM with different temperatures. For the PCM only system, the generated vortices due to natural convection start to join and become larger, a phenomenon also established in the literature [64]. After the melting process, a lower temperature gradient happens in the presence of porous medium and the magnitude of minimum temperature is much higher than PCM only since there is still solid PCM at the initial temperature in the domain.

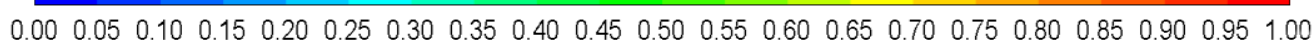

$\begin{array}{llll}100 \mathrm{~s} & 300 \mathrm{~s} & 500 \mathrm{~s} & 700 \mathrm{~s}\end{array}$



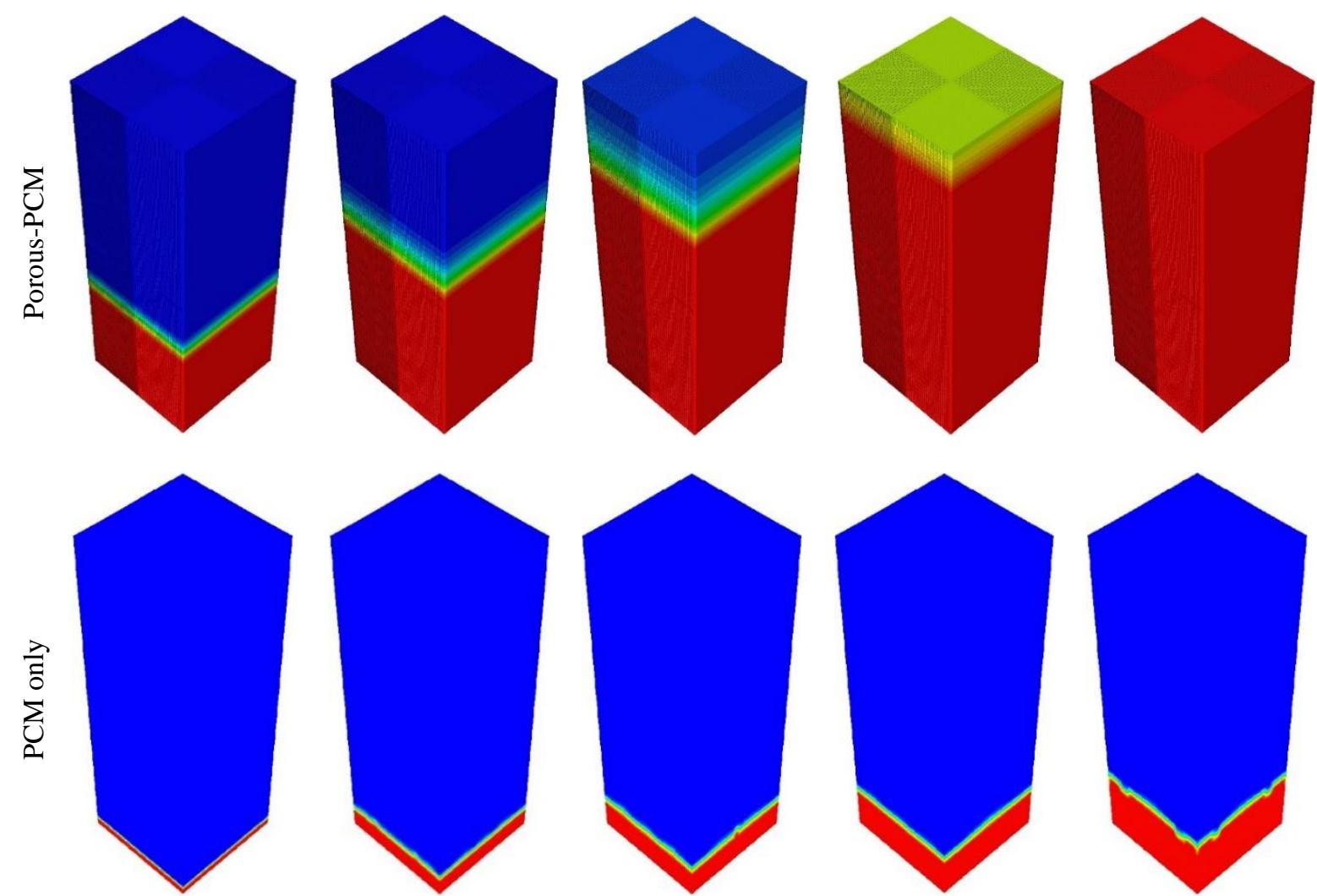

Fig. 4. The contour plot of liquid fraction at different times for the porous-PCM case compared with PCM only

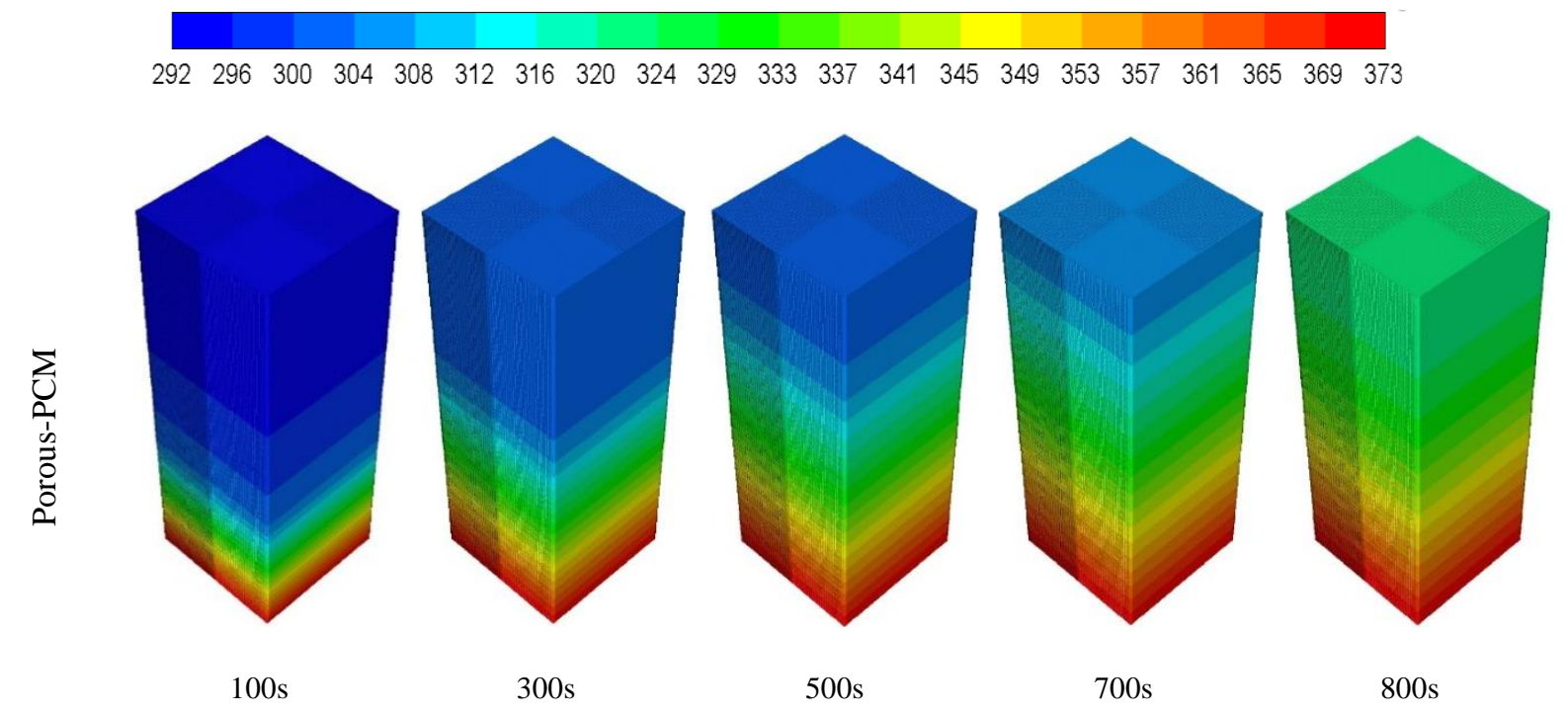



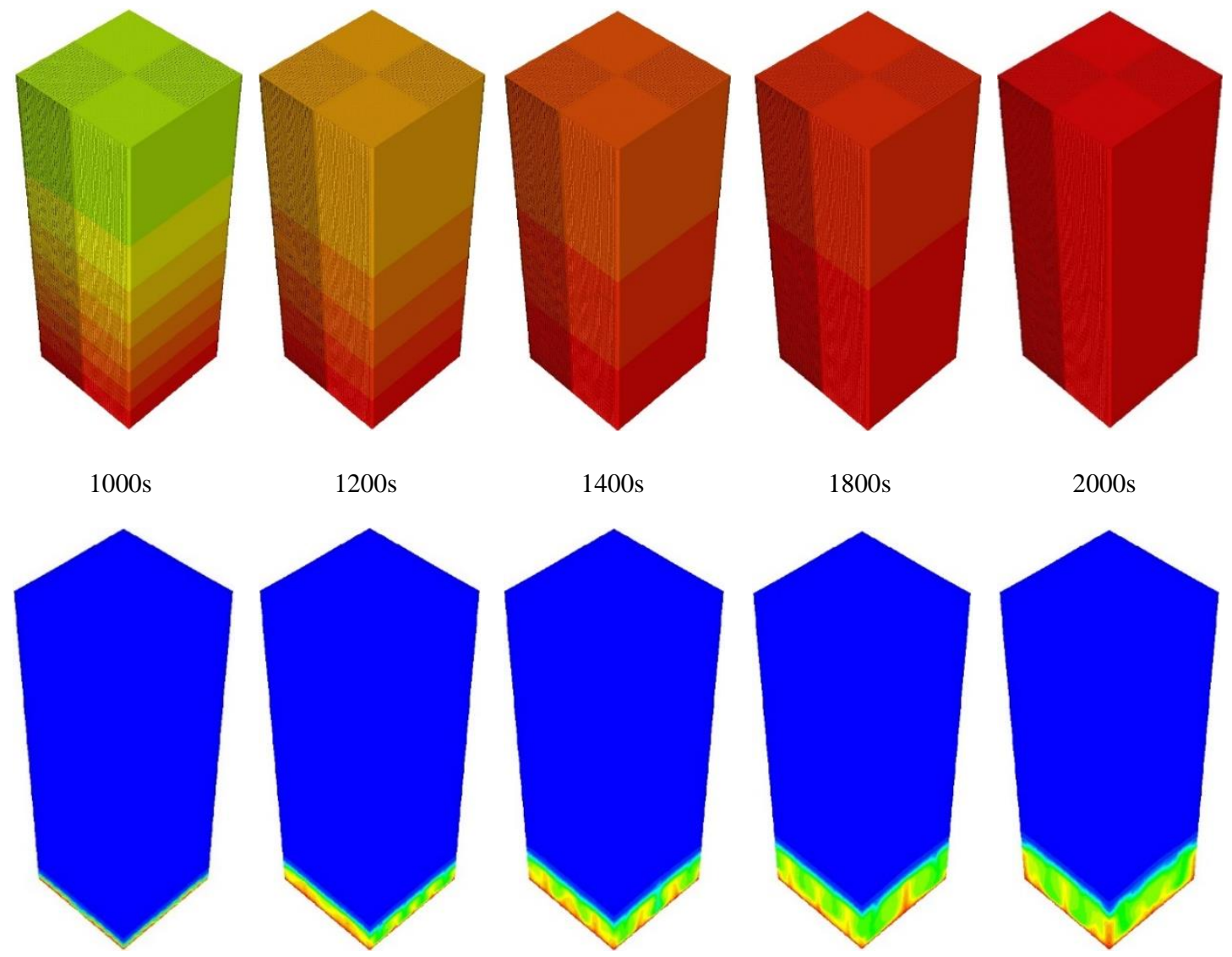

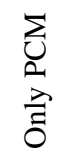

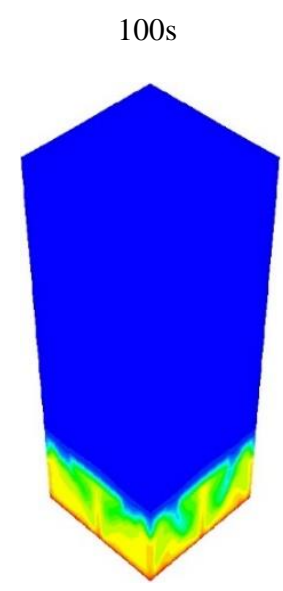

$1000 \mathrm{~s}$

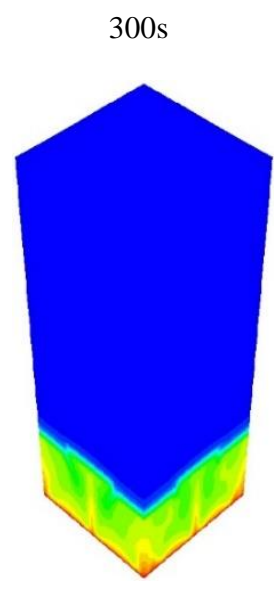

1200 s

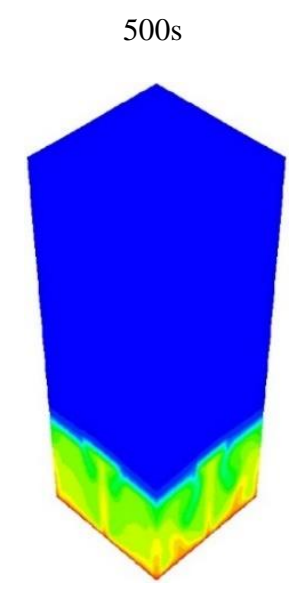

$1400 s$

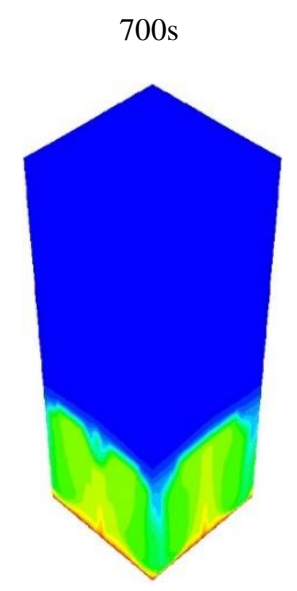

$1800 \mathrm{~s}$

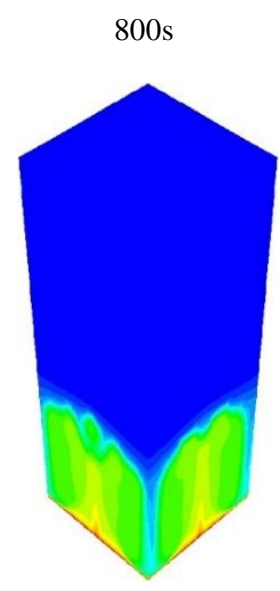

$2000 \mathrm{~s}$

Fig. 5. The contour plot of temperature distribution at different times for the porous-PCM case compared with PCM only

To better understand the PCM only system, Fig. 6 shows the distribution of liquid fraction and temperature at different times for the PCM only system after $2000 \mathrm{~s}$ until the end of the melting process which is $5174 \mathrm{~s}$. With the elapse of time, the effect of natural convection increases in 
the domain and becomes dominant and therefore by circulating of the liquid PCM, the rate of heat transfer increases which helps the heat storage process.

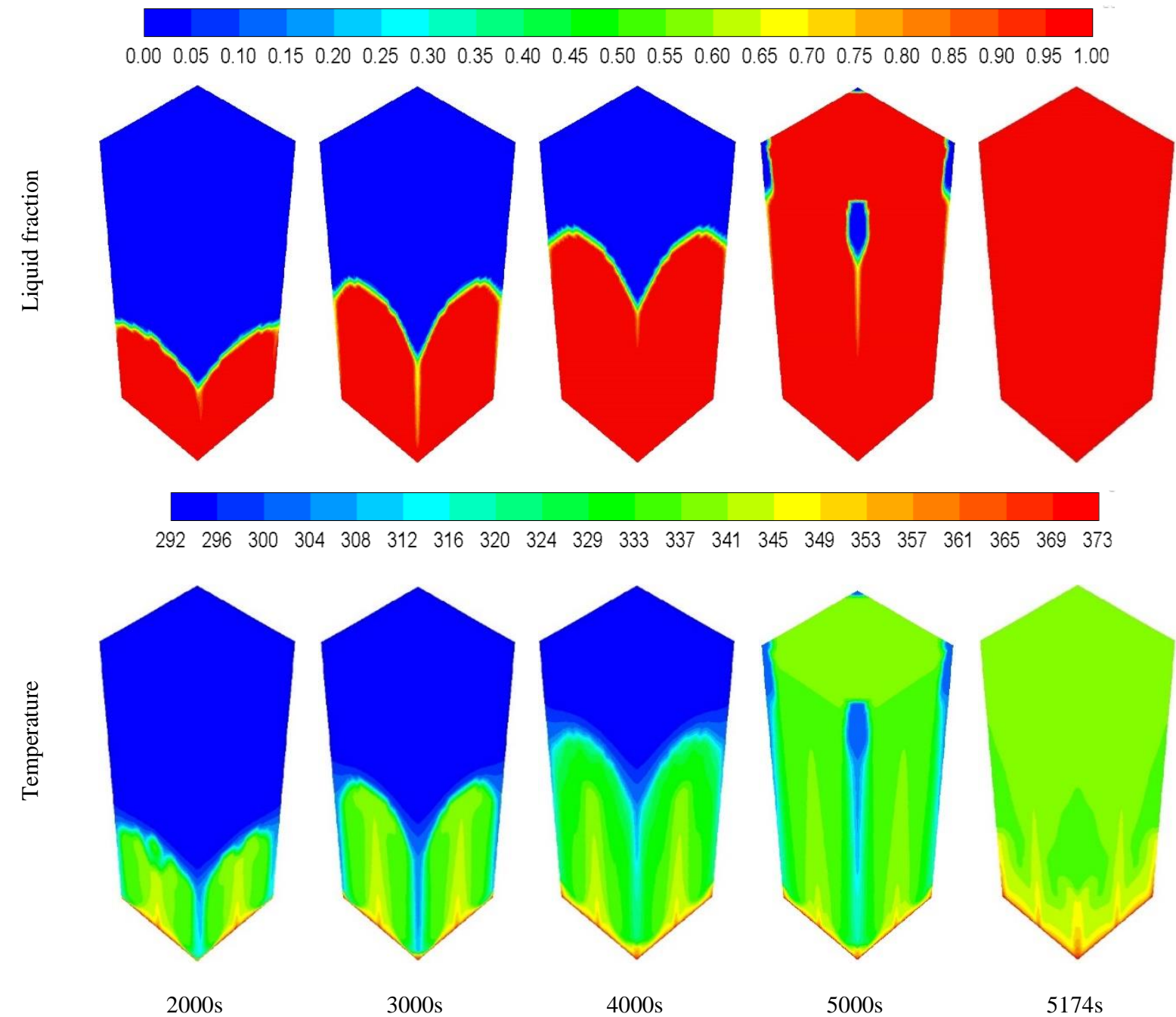

Fig. 6. The contour plot of temperature distribution at different times for the porous-PCM case compared with only PCM

The variation of average PCM liquid fraction and temperature as a function of time is shown in Figs. 7-a and 7-b, respectively. The temperature of PCM in the presence of the porous medium is higher than the PCM only system, causing a higher liquid fraction at all times. By using the porous medium inside the PCM, the melting time reduces by almost $85 \%$ compared with the only PCM system. 


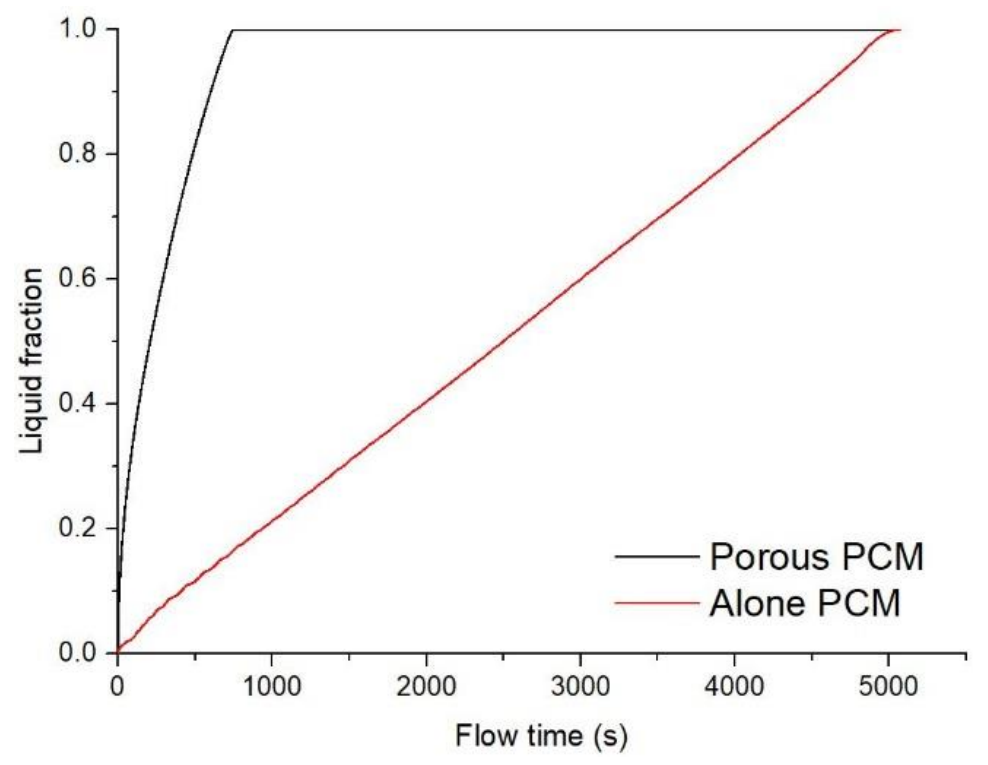

a)

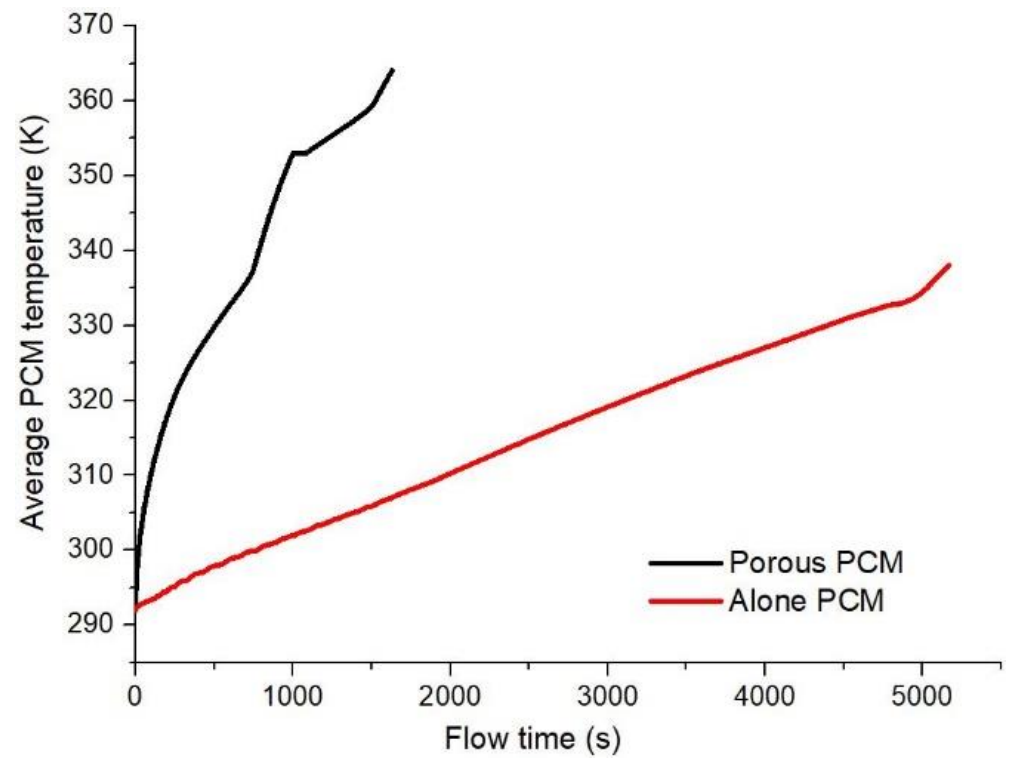

b)

Fig. 7. The variation of mean a) Liquid fraction and b) temperature as a function of time for the porous-PCM compared with the PCM only

Fig. 8 presents the temperature at different heights for both systems of porous-PCM and PCM only at the centre of the system. For the PCM only system, the temperature remains at the initial temperature $(292 \mathrm{~K})$ until the melting front reaches the point; however, for the porous- PCM, due to the high rate of heat transfer, the temperatures at different heights rise from the beginning. The temperatures at the height of 7.5 and $12.5 \mathrm{~cm}$ rise after $1800 \mathrm{~s}$ and $3440 \mathrm{~s}$, 
respectively, in the PCM only case. Due to the natural convection effect and recirculation of the flow, a fluctuation is observed in the temperature of each point.

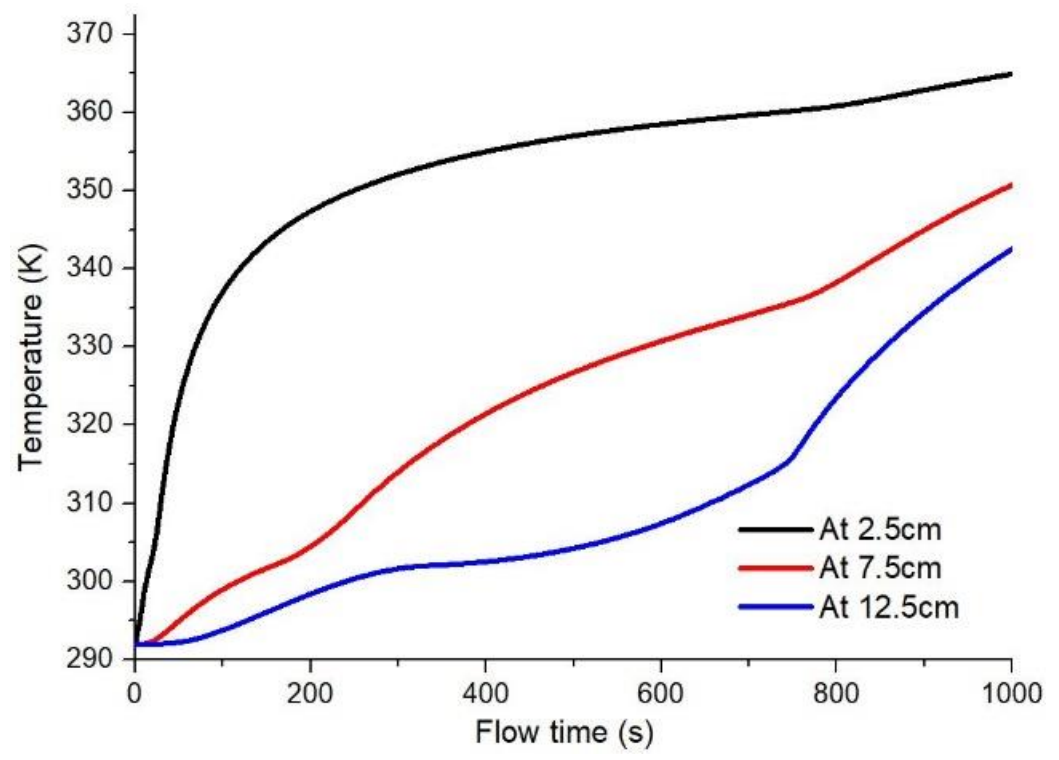

a)

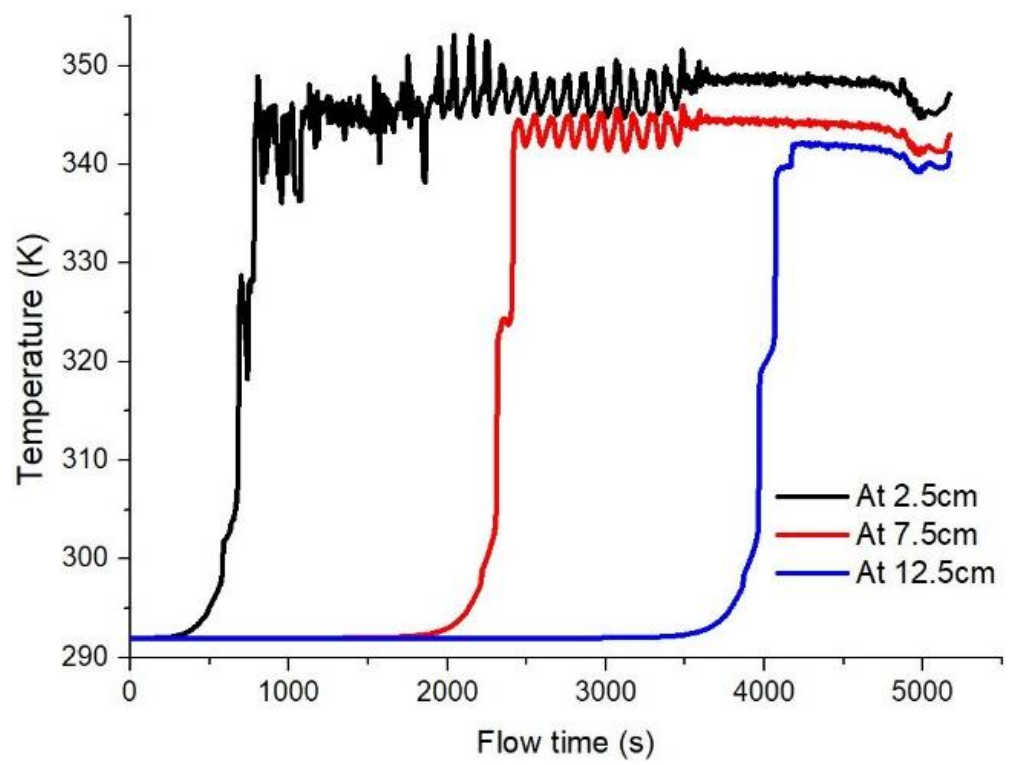

b)

Fig. 8. The variation of temperature at different heights as a function of time for the cases of a) the porous-PCM and b) the PCM only 


\subsection{Effect of porosity}

By reducing the porosity of the metal foam and as a result increasing the amount of high conductivity metal in the system, a higher rate of heat transfer is achieved as expected. Therefore, the PCM melts in a shorter time. However, it is not recommended due to the consequent reduction of the mass of PCM in the system which is the main volume of heat storage [32]. Figs. 9-a and 9-b illustrate the variation of liquid fraction as well as temperature as a function of time for different porosity of the metal foam. By increasing the portion of metal foams from porosity of 0.95 to 0.9 and 0.85 , the melting time reduces by $50.7 \%$ and $67.9 \%$, respectively. As shown in Fig. 9-b, at an identical time, the temperature increases faster for lower porosity.

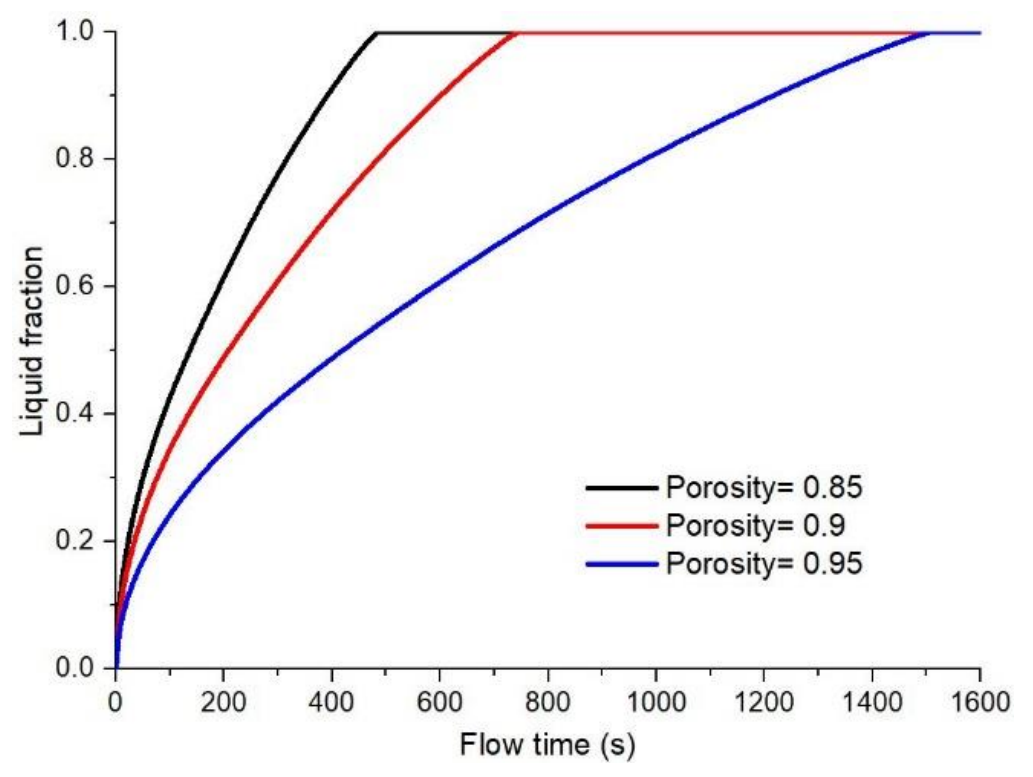

a) 


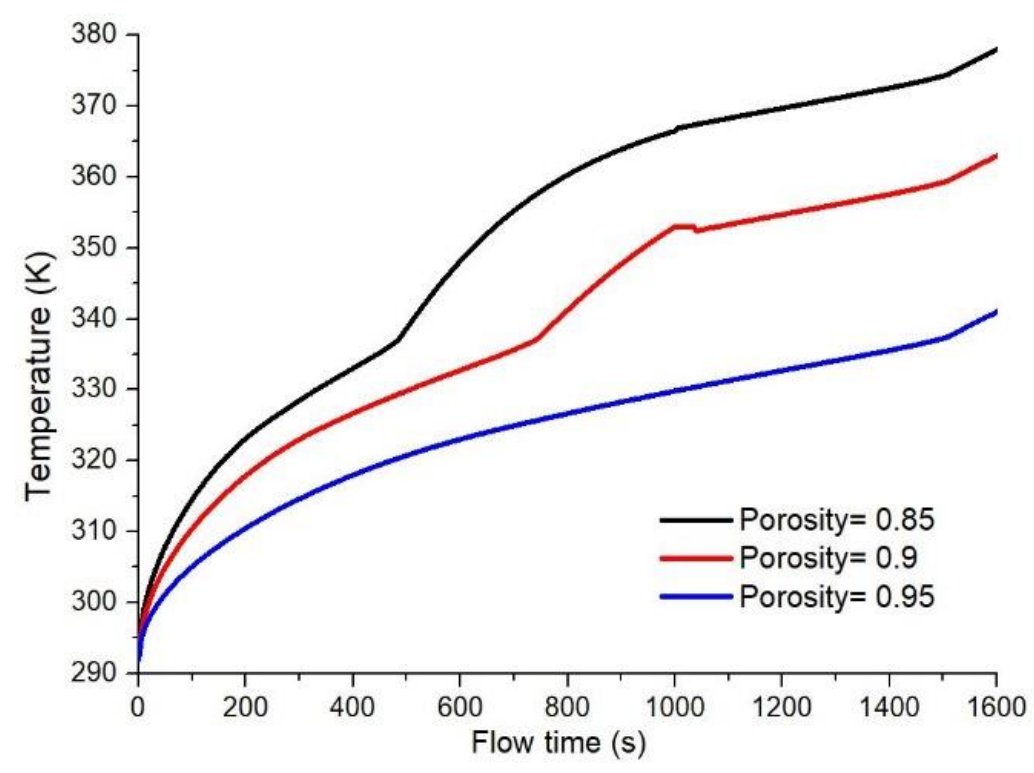

b)

Fig. 9. The variation of mean a) Liquid fraction and b) temperature as a function of time for the porous-PCM system for different porosity

\subsection{Effect of pore density}

Fig. 10 shows the effect of pore density on the liquid fraction and temperature variation of the porous PCM LHS unit. The pore density has a negligible effect on the temperature profile inside the domain and therefore there is no considerable change happens for a different pore density of the metal foam which was also indicated in the literature [50]. Therefore, the melting time is not changed for a different pore density of the metal foam.

The reason is that since the porosity is considered constant, the amount of metal used in the unit is constant. For example, for the case of 10 PPI compared with 50 PPI, lower number of pores are placed in the unit length of the system. However, to have a constant porosity, the metal thickness between to pores should be higher for the case of 10 PPI compared with 50 PPI which cause a higher effective thermal conductivity. On the other hand, for the case of 50 PPI, the PCM is divided by a higher number of such an encapsulation which results in higher performance compared with 10 PPI case. As shown, one positive effect and one negative effect of pore density results in an almost negligible effect on the temperature and liquid fraction. 


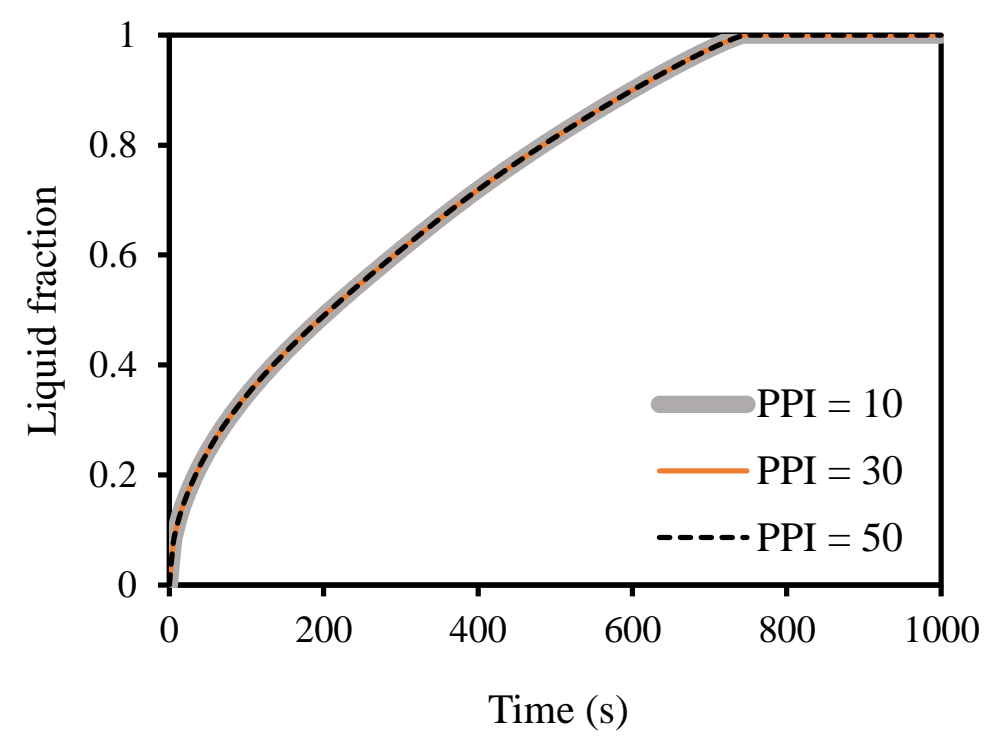

a)

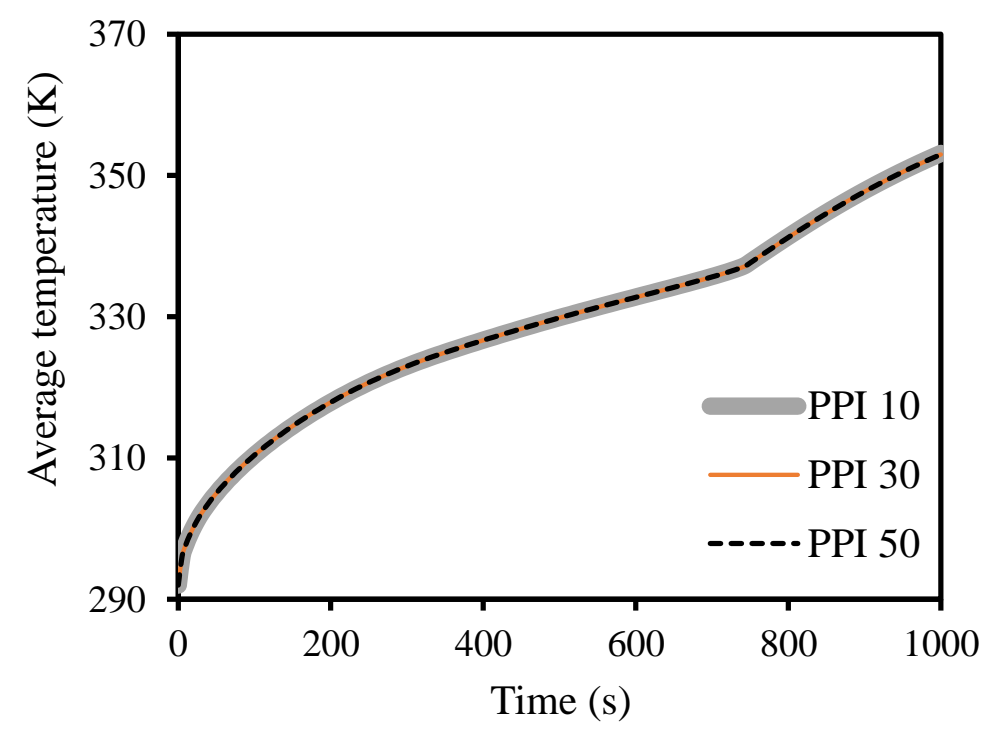

b)

Fig. 10. The variation of mean a) Liquid fraction and b) temperature as a function of time for the porous-PCM system for different pore densities of the metal foam

\subsection{Effect of thermal model: Non-equilibrium vs Equilibrium}

Two different thermal models are available to solve the energy equation, the non-equilibrium model, which considers the heat transfer between the liquid phase and the solid porous medium 
and the equilibrium model which simplifies the energy equation by considering the temperature of the liquid PCM and porous medium to be the same, as for a homogeneous mixture. The results of the non-equilibrium model are much closer to reality than the equilibrium model. As shown in the validation section, especially after the liquidus temperature, the thermal equilibrium model cannot predict the temperature distribution very well and the disagreement enhances for a higher temperature. However, in phase change materials, since the operating temperature range is highly localised at the melting point, the results of the two different models might be close. Figs. 11-a and 11-b illustrate the variation of liquid fraction and temperature as a function of time for the two models. As shown in the temperature profile, the difference between the two models is more related to the time post melting only. During the melting process, the temperatures based on both models are almost the same which is also shown in Fig. 3. As shown, the melting time is a little higher for the non-equilibrium model compared with the equilibrium one.

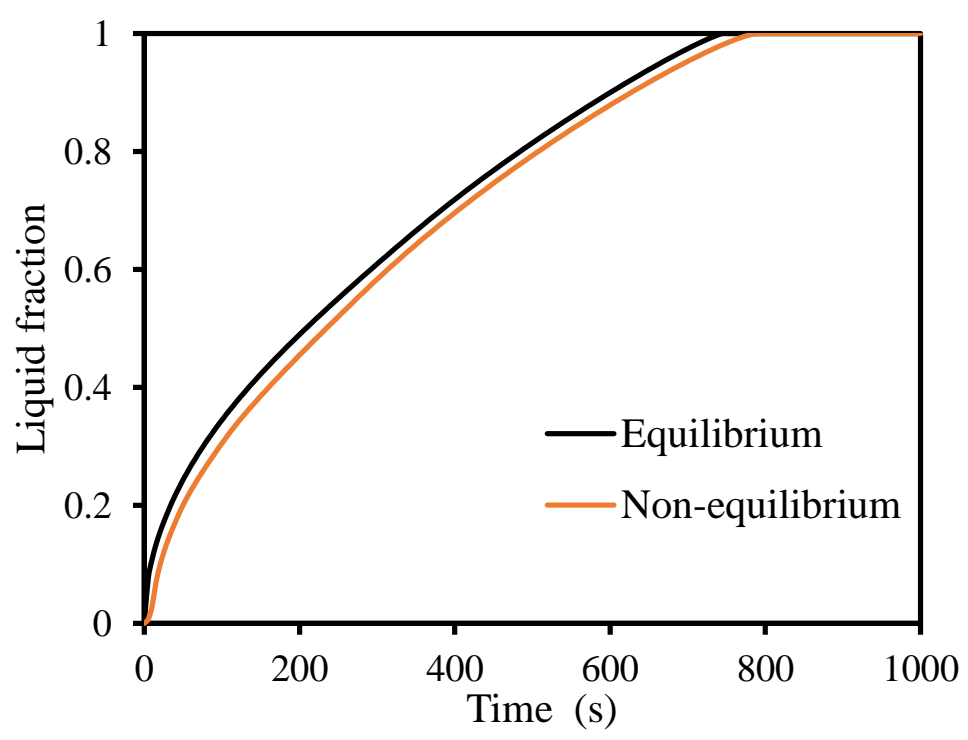

a) 


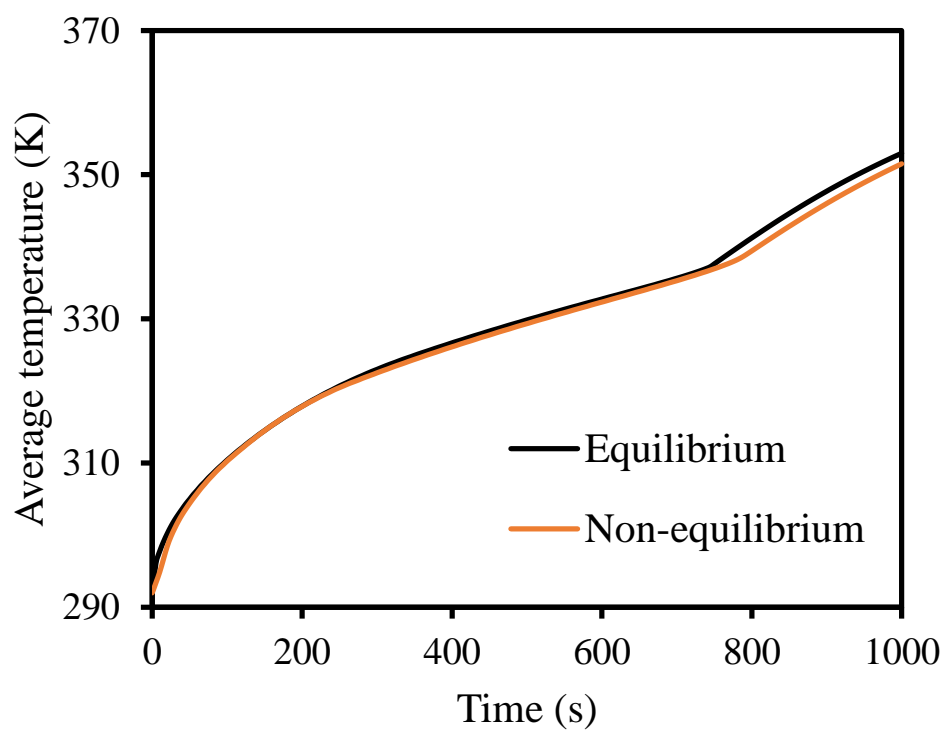

b)

Fig. 11. The variation of mean a) Liquid fraction and b) temperature as a function of time for the porous-PCM system for different thermal models

\subsection{Effect of heater location}

Three different locations are considered for the heater in this study including the entire surface of the bottom and top as well as the same area in the side wall as shown in Fig. 1. For the top and bottom heaters, due to the effect of gravity and natural convection, the location of the heater affects the melting process. However, in the porous-PCM cases, due to the presence of the porous medium which makes a flow resistance and therefore suppressing the effect of natural convection, the effect of the heater location is expected to be low. For the side heater, since the type of heating element is a constant temperature heater in the study, it can spread out the heat from the middle of the domain better than the top or bottom heaters. In other words, by locating the heater at the middle of the unit, the heat can be transferred to all the domain faster. Figs. 12-a and 12-b illustrate the effect of heater location on the liquid fraction and average temperature as a function of time. Both liquid fraction and temperature almost coincide to each other for the heater located at the top and bottom of the domain due to the mentioned reason. 
The melting time decreases by only $4.7 \%$ for the top heater compared with the bottom heater due to small effect of natural convection. For the side heater, the melting time decreases by almost $70.5 \%$ compared with that for the bottom heater. As shown in Fig. 12-b, high average temperature of PCM for the side heating can be shown compared with the bottom or side heating. After the time of $220 \mathrm{~s}$ when all the PCM melts for the side heating case, the mean temperature rises sharply since the PCM gains all the required latent heat and places in the sensible heat section. After almost $800 \mathrm{~s}$, the PCM average temperature almost reaches to the heater temperature for the side heating case.

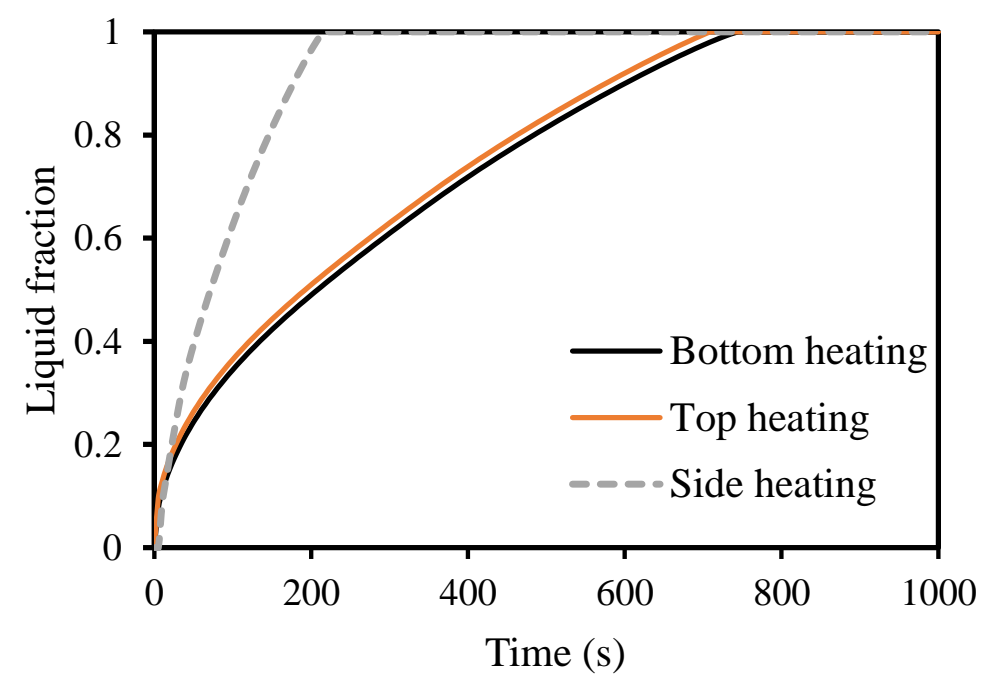

a) 


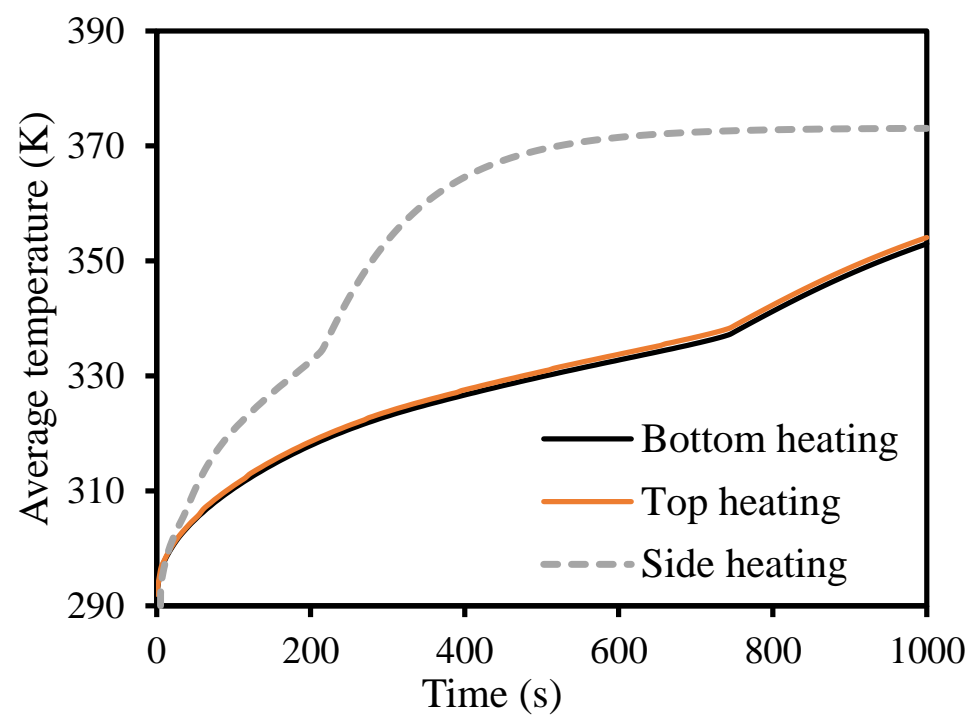

b)

Fig. 12. The variation of mean a) Liquid fraction and b) temperature as a function of time for the porous-PCM system for different locations of the heater

\subsection{Effect of multiple-segment metal foam instead of one}

By using a porous material in the latent heat storage systems, in one hand, heat transfer rate inside the PCM enhances resulting in a shorter melting time which is more effective by reducing the porosity. On the other hand, it reduces the total mass of the PCM in the unit which would be otherwise replaced by the PCM which stores a higher amount of heat. Therefore, high porosity is always recommended. In this study, instead of using a porous material with the porosity of $90 \%$ in the whole domain, three different porous media are employed with the porosity of $85 \%$ at the bottom, $90 \%$ in the middle and $95 \%$ at the top for the heater located at the bottom of the system. The reason for using the least porosity at the bottom is that this section is connected to the heat source and therefore the foam with a lower porosity can transfer the heat faster from the heater to all the domain. 
Fig. 13 illustrates the contour plot of liquid fraction and temperature at different times using the multiple-segment porous medium which can be compared with the results of uniform porosity of $90 \%$ in Fig. 4 for the liquid fraction and Fig. 5 for the temperature. Higher values of the liquid fraction can be seen for the multiple-segment unit compared with the uniform case.

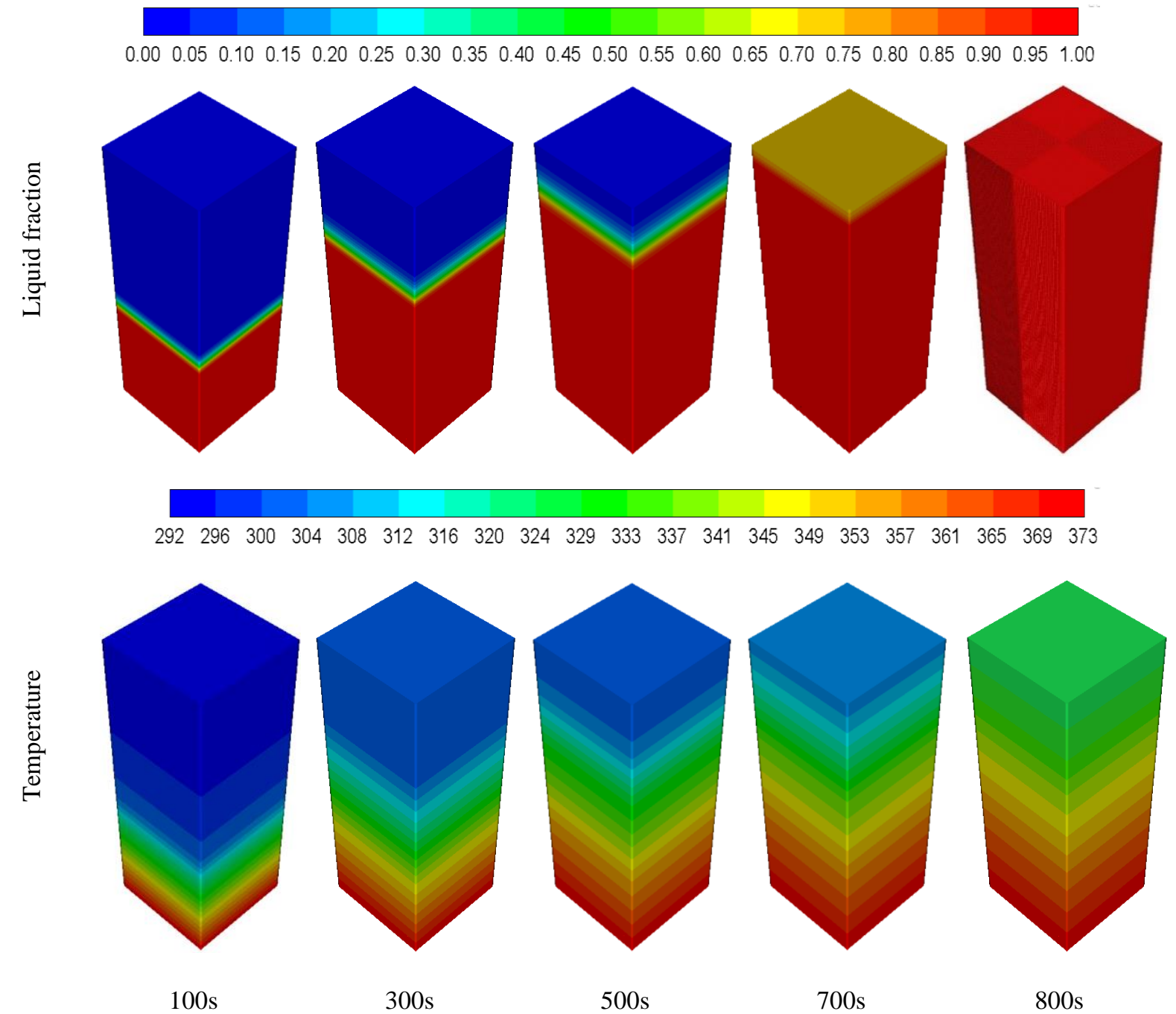

Fig. 13. The contour plot of liquid fraction and the temperature distribution in case of multiple-segments metal foam

Figs.14-a and 14-b illustrate the effect of using the multiple-segment porous medium on the mean liquid fraction and temperature variation with time. A higher average temperature of the 
PCM can be seen for the constant porosity case compared with the multiple-segment unit; however, the average melting time reduces by $3.5 \%$. At the beginning, the liquid fraction of the multiple-segment unit is lower than the constant porosity case since the mass of PCM at the bottom of the storage unit for the multiple-segment system is less than that for the uniform porous system and the heat is more transferred by the porous medium rather than storing in the PCM. However, at the end of the process, higher values of average liquid fraction can be seen for an identical time.

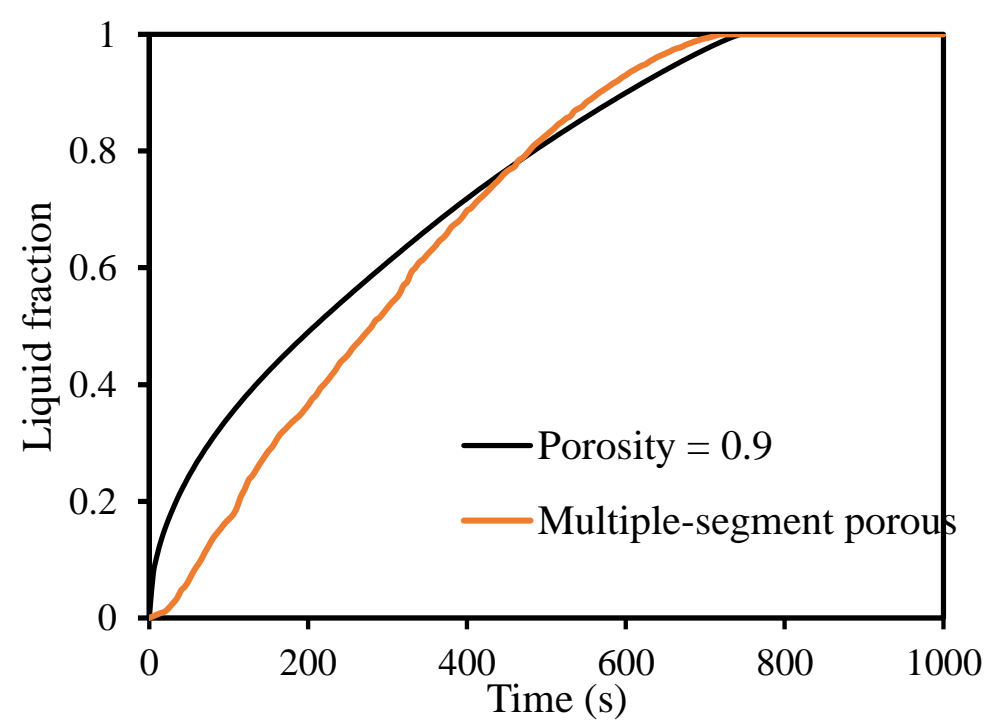

a) 


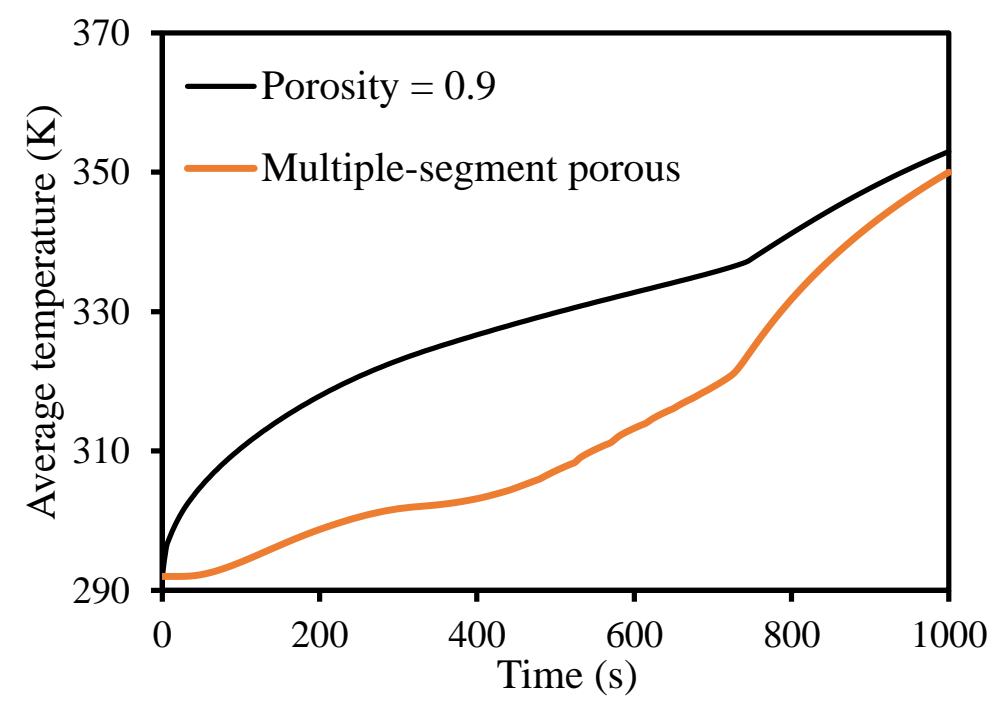

b)

Fig. 14. The variation of mean a) Liquid fraction and b) temperature as a function of time for the multiple-segment porous-PCM system compared with the uniform porous system

To better understand the effect of the multiple-segment porous unit, Fig. 15 displays the variation of temperature at different points in different zones of the domain. The temperature at the height of $2.5 \mathrm{~cm}$ rises rapidly due to the low porosity of the foam in the bottom layer as well as the small distance from the heat source. As shown, the rate of temperature enhancement reduces by increasing the porosity at each layer. 


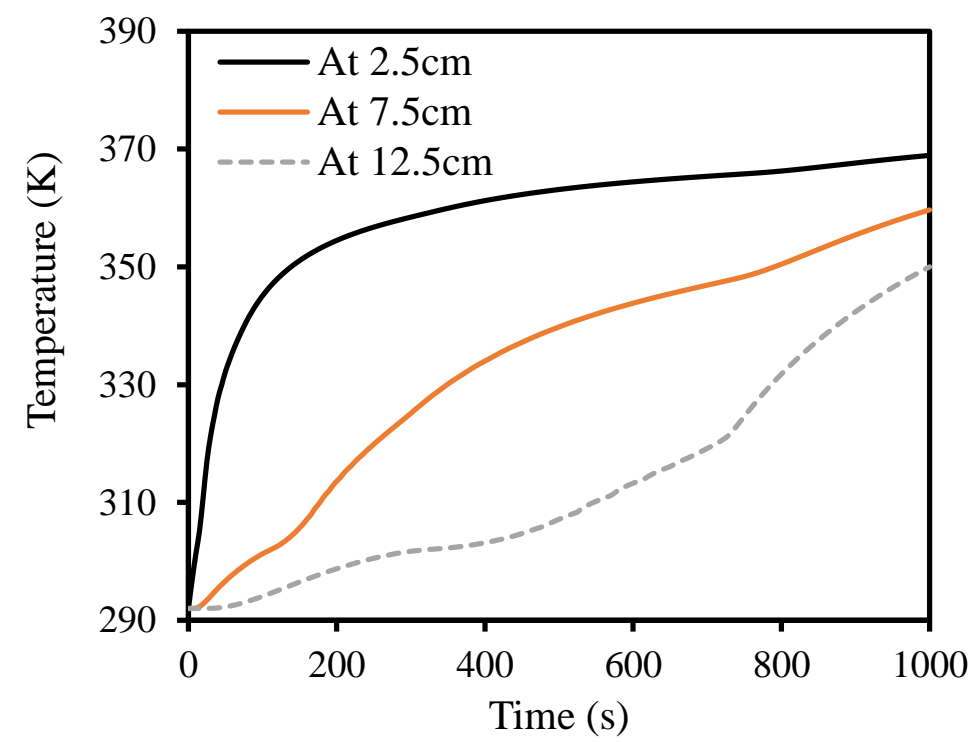

Fig. 15. The variation of temperature at different heights as a function of time for the multiple-segment porous case

This study shows that the composition of the low porosity porous-PCM composite with multiple-segment porous structure gives better performance (regarding the heat transfer and melting time) of the phase change process in the latent heat storage systems.

\section{Conclusion}

Phase change material in the thermal energy storage unit has fairly low thermal property values which affects negatively on the energy system performance. This study aimed to find the optimal values of different design variables to enhance the performance of the system. The effects of copper foam porosity and pore size, non-equilibrium porous medium model case, multiple-porous segment system and different heater locations in the system were studied on the heat transfer performance including the liquid fraction and temperature in a vertical rectangular container. The numerical finding was validated by experimental data. After showing the advantages of porous-PCM, it can be concluded that providing the porous foam accelerates the melting rate and the low porosity shows better performance than the high porosity with no effects registered by changing the pore size. The use of multiple-segment 
porous structure instead of the uniform one shows a reduction of $3.5 \%$ in the melting time. The porous-PCM composite shows an $85 \%$ reduction in the melting time compared with the PCM only system. A more accurate non-equilibrium model shows $7.4 \%$ difference in melting time compared with the equilibrium model. By changing the location of the constant temperature heater from the bottom to the side and top surface, the melting time decreases by $70.5 \%$ and $4.7 \%$, respectively. The results of this paper provide guidelines to develop more efficient LHS unit for the practical application of a high-performance unit.

\section{References}

[1] Miró L, Gasia J, Cabeza LF. Thermal energy storage (TES) for industrial waste heat (IWH) recovery: A review. Applied energy. 2016;179:284-301.

[2] Sharma A, Tyagi VV, Chen CR, Buddhi D. Review on thermal energy storage with phase change materials and applications. Renewable and Sustainable Energy Reviews. $2009 ; 13(2): 318-45$.

[3] Neeper DA. Thermal dynamics of wallboard with latent heat storage. Solar Energy. 2000;68(5):393-403.

[4] Medina MA, King JB, Zhang M. On the heat transfer rate reduction of structural insulated panels (SIPs) outfitted with phase change materials (PCMs). Energy. 2008;33(4):667-78.

[5] Buddhi D. Thermal performance of a shell and tube PCM storage heat exchanger for industrial waste heat recovery. presented at solar world congress, Taejon, Korea, August 24th30th. 1997.

[6] Mettawee E-BS, Assassa GMR. Experimental study of a compact PCM solar collector. Energy. 2006;31(14):2958-68.

[7] Michels H, Pitz-Paal R. Cascaded latent heat storage for parabolic trough solar power plants. Solar Energy. 2007;81(6):829-37. 
[8] El-Sebaii AA, Al-Ghamdi AA, Al-Hazmi FS, Faidah AS. Thermal performance of a single basin solar still with PCM as a storage medium. Applied Energy. 2009;86(7):1187-95.

[9] Tyagi VV, Buddhi D. Thermal cycle testing of calcium chloride hexahydrate as a possible PCM for latent heat storage. Solar Energy Materials and Solar Cells. 2008;92(8):891-9.

[10] El-Sebaii AA, Al-Heniti S, Al-Agel F, Al-Ghamdi AA, Al-Marzouki F. One thousand thermal cycles of magnesium chloride hexahydrate as a promising PCM for indoor solar cooking. Energy Conversion and Management. 2011;52(4):1771-7.

[11] Tian Y, Zhao CY. A numerical investigation of heat transfer in phase change materials (PCMs) embedded in porous metals. Energy. 2011;36(9):5539-46.

[12] Ettouney HM, Alatiqi I, Al-Sahali M, Ahmad Al-Ali S. Heat transfer enhancement by metal screens and metal spheres in phase change energy storage systems. Renewable Energy. 2004;29(6):841-60.

[13] Xiao X, Zhang P, Li M. Preparation and thermal characterization of paraffin/metal foam composite phase change material. Applied Energy. 2013;112:1357-66.

[14] Warzoha RJ, Weigand RM, Fleischer AS. Temperature-dependent thermal properties of a paraffin phase change material embedded with herringbone style graphite nanofibers. Applied Energy. 2015;137:716-25.

[15] Seeniraj RV, Velraj R, Lakshmi Narasimhan N. Thermal analysis of a finned-tube LHTS module for a solar dynamic power system. Heat and Mass Transfer. 2002;38(4):409-17.

[16] Agyenim F, Eames P, Smyth M. Experimental study on the melting and solidification behaviour of a medium temperature phase change storage material (Erythritol) system augmented with fins to power a $\mathrm{LiBr} / \mathrm{H} 2 \mathrm{O}$ absorption cooling system. Renewable Energy. 2011;36(1):108-17.

[17] Tay N, Belusko M, Bruno F. Experimental investigation of tubes in a phase change thermal energy storage system. Applied energy. 2012;90(1):288-97. 
[18] Shahsavar A, Al-Rashed AAAA, Entezari S, Sardari PT. Melting and solidification characteristics of a double-pipe latent heat storage system with sinusoidal wavy channels embedded in a porous medium. Energy. 2019;171:751-69.

[19] Medrano M, Yilmaz M, Nogués M, Martorell I, Roca J, Cabeza LF. Experimental evaluation of commercial heat exchangers for use as PCM thermal storage systems. Applied energy. 2009;86(10):2047-55.

[20] Stritih U. An experimental study of enhanced heat transfer in rectangular PCM thermal storage. International Journal of Heat and Mass Transfer. 2004;47(12-13):2841-7.

[21] Mahdi JM, Lohrasbi S, Ganji DD, Nsofor EC. Accelerated melting of PCM in energy storage systems via novel configuration of fins in the triplex-tube heat exchanger. International Journal of Heat and Mass Transfer. 2018;124:663-76.

[22] Mahdi JM, Nsofor EC. Solidification enhancement of PCM in a triplex-tube thermal energy storage system with nanoparticles and fins. Applied Energy. 2018;211:975-86.

[23] Singh RP, Kaushik SC, Rakshit D. Melting phenomenon in a finned thermal storage system with graphene nano-plates for medium temperature applications. Energy Conversion and Management. 2018;163:86-99.

[24] Huang Z, Gao X, Xu T, Fang Y, Zhang Z. Thermal property measurement and heat storage analysis of $\mathrm{LiNO} 3 / \mathrm{KCl}$ - expanded graphite composite phase change material. Applied Energy. 2014;115:265-71.

[25] Li Z, Sun WG, Wang G, Wu ZG. Experimental and numerical study on the effective thermal conductivity of paraffin/expanded graphite composite. Solar Energy Materials and Solar Cells. 2014;128:447-55.

[26] Merlin K, Soto J, Delaunay D, Traonvouez L. Industrial waste heat recovery using an enhanced conductivity latent heat thermal energy storage. Applied Energy. 2016;183:491-503. 
[27] Li Y, Guo B, Huang G, Kubo S, Shu P. Characterization and thermal performance of nitrate mixture/SiC ceramic honeycomb composite phase change materials for thermal energy storage. Applied Thermal Engineering. 2015;81:193-7.

[28] Diao Y, Kang Y, Liang L, Zhao Y, Zhu T. Experimental investigation on the heat transfer performance of a latent thermal energy storage device based on flat miniature heat pipe arrays. Energy. 2017;138:929-41.

[29] Lohrasbi S, Miry SZ, Gorji-Bandpy M, Ganji DD. Performance enhancement of finned heat pipe assisted latent heat thermal energy storage system in the presence of nano-enhanced H2O as phase change material. International Journal of Hydrogen Energy. 2017;42(10):652646.

[30] Atal A, Wang Y, Harsha M, Sengupta S. Effect of porosity of conducting matrix on a phase change energy storage device. International Journal of Heat and Mass Transfer. 2016;93:9-16.

[31] Wang H, Wang F, Li Z, Tang Y, Yu B, Yuan W. Experimental investigation on the thermal performance of a heat sink filled with porous metal fiber sintered felt/paraffin composite phase change material. Applied Energy. 2016;176:221-32.

[32] Mahdi JM, Nsofor EC. Solidification enhancement in a triplex-tube latent heat energy storage system using nanoparticles-metal foam combination. Energy. 2017;126:501-12.

[33] Wu Z, Zhao C. Experimental investigations of porous materials in high temperature thermal energy storage systems. Solar Energy. 2011;85(7):1371-80.

[34] Tao YB, Lin CH, He YL. Preparation and thermal properties characterization of carbonate salt/carbon nanomaterial composite phase change material. Energy Conversion and Management. 2015;97:103-10.

[35] Mahdi JM, Nsofor EC. Solidification of a PCM with nanoparticles in triplex-tube thermal energy storage system. Applied Thermal Engineering. 2016;108:596-604. 
[36] Huo Y, Rao Z. Lattice Boltzmann investigation on phase change of nanoparticle-enhanced phase change material in a cavity with separate plate. Energy Conversion and Management. 2017;154:420-9.

[37] Wang F, Zhang C, Liu J, Fang X, Zhang Z. Highly stable graphite nanoparticle-dispersed phase change emulsions with little supercooling and high thermal conductivity for cold energy storage. Applied Energy. 2017;188:97-106.

[38] Zhao CY, Lu W, Tian Y. Heat transfer enhancement for thermal energy storage using metal foams embedded within phase change materials (PCMs). Solar Energy. 2010;84(8):1402-12.

[39] Xu Y, Ren Q, Zheng Z-J, He Y-L. Evaluation and optimization of melting performance for a latent heat thermal energy storage unit partially filled with porous media. Applied Energy. 2017;193:84-95.

[40] Lafdi K, Mesalhy O, Shaikh S. Experimental study on the influence of foam porosity and pore size on the melting of phase change materials. Journal of Applied Physics. 2007;102(8):083549.

[41] Nithyanandam K, Pitchumani R. Computational Studies on Metal Foam and Heat Pipe Enhanced Latent Thermal Energy Storage. Journal of Heat Transfer. 2014;136(5):051503--10. [42] Zhang P, Xiao X, Meng ZN, Li M. Heat transfer characteristics of a molten-salt thermal energy storage unit with and without heat transfer enhancement. Applied Energy. $2015 ; 137: 758-72$.

[43] Yang X, Wang W, Yang C, Jin L, Lu TJ. Solidification of fluid saturated in open-cell metallic foams with graded morphologies. International Journal of Heat and Mass Transfer. 2016;98:60-9. 
[44] Zhu F, Zhang C, Gong X. Numerical analysis on the energy storage efficiency of phase change material embedded in finned metal foam with graded porosity. Applied Thermal Engineering. 2017; 123:256-65.

[45] Tao YB, You Y, He YL. Lattice Boltzmann simulation on phase change heat transfer in metal foams/paraffin composite phase change material. Applied Thermal Engineering. 2016;93:476-85.

[46] Wang P, Yao H, Lan Z, Peng Z, Huang Y, Ding Y. Numerical investigation of PCM melting process in sleeve tube with internal fins. Energy Conversion and Management. 2016;110:428-35.

[47] Tao YB, He YL. Effects of natural convection on latent heat storage performance of salt in a horizontal concentric tube. Applied Energy. 2015;143:38-46.

[48] Nield DA, Bejan A. Convection in Porous Media: Springer International Publishing, 2017. [49] Mahdi JM, Nsofor EC. Melting enhancement in triplex-tube latent heat energy storage system using nanoparticles-metal foam combination. Applied Energy. 2017;191:22-34.

[50] Liu Z, Yao Y, Wu H. Numerical modeling for solid-liquid phase change phenomena in porous media: Shell-and-tube type latent heat thermal energy storage. Applied Energy. 2013;112:1222-32.

[51] Yang H, Zhao M, Gu ZL, Jin LW, Chai JC. A further discussion on the effective thermal conductivity of metal foam: An improved model. International Journal of Heat and Mass Transfer. 2015;86:207-11.

[52] Boomsma K, Poulikakos D. On the effective thermal conductivity of a threedimensionally structured fluid-saturated metal foam. International Journal of Heat and Mass Transfer. 2001;44(4):827-36. 
[53] Dai Z, Nawaz K, Park YG, Bock J, Jacobi AM. Correcting and extending the BoomsmaPoulikakos effective thermal conductivity model for three-dimensional, fluid-saturated metal foams. International Communications in Heat and Mass Transfer. 2010;37(6):575-80.

[54] Calmidi VV, Mahajan RL. The Effective Thermal Conductivity of High Porosity Fibrous Metal Foams. Journal of Heat Transfer. 1999;121(2):466-71.

[55] Bhattacharya A, Calmidi VV, Mahajan RL. Thermophysical properties of high porosity metal foams. International Journal of Heat and Mass Transfer. 2002;45(5):1017-31.

[56] Boomsma K, Poulikakos D. Corrigendum for the paper: K. Boomsma, D. Poulikakos, "On the effective thermal conductivity of a three-dimensionally structured fluid-saturated metal foam" [International Journal of Heat and Mass Transfer, 44 (2001) 827-836]. International Journal of Heat and Mass Transfer. 2011;54(1):746-8.

[57] Žukauskas A. Heat Transfer from Tubes in Crossflow. In: Hartnett JP, Irvine TF, editors. Advances in Heat Transfer: Elsevier; 1972. p. 93-160.

[58] Al-Abidi AA, Mat S, Sopian K, Sulaiman MY, Mohammad AT. Internal and external fin heat transfer enhancement technique for latent heat thermal energy storage in triplex tube heat exchangers. Applied Thermal Engineering. 2013;53(1):147-56.

[59] Wang P, Wang X, Huang Y, Li C, Peng Z, Ding Y. Thermal energy charging behaviour of a heat exchange device with a zigzag plate configuration containing multi-phase-changematerials (m-PCMs). Applied Energy. 2015;142:328-36.

[60] Shahsavar A, Shaham A, Talebizadehsardari P. Wavy channels triple-tube LHS unit with sinusoidal variable wavelength in charging/discharging mechanism. International Communications in Heat and Mass Transfer. 2019;107:93-105.

[61] Mat S, Al-Abidi AA, Sopian K, Sulaiman MY, Mohammad AT. Enhance heat transfer for PCM melting in triplex tube with internal-external fins. Energy Conversion and Management. 2013;74:223-36. 
[62] Ye W-B, Zhu D-S, Wang N. Numerical simulation on phase-change thermal storage/release in a plate-fin unit. Applied Thermal Engineering. 2011;31(17):3871-84.

[63] Assis E, Katsman L, Ziskind G, Letan R. Numerical and experimental study of melting in a spherical shell. International Journal of Heat and Mass Transfer. 2007;50(9):1790-804.

[64] Darzi AR, Farhadi M, Sedighi K. Numerical study of melting inside concentric and eccentric horizontal annulus. Applied Mathematical Modelling. 2012;36(9):4080-6. 